\title{
Seeded Heteroepitaxial Growth of Crystallizable Collagen Triple Helices: Engineering Multifunctional Two-Dimensional Core-Shell Nanostructures
}

Andrea D. Merg, ${ }^{\dagger}$ Eric van Genderen, ${ }^{\ddagger}$ Alisina Bazrafshan,${ }^{\dagger}$ Hanquan Su, ${ }^{\dagger}$ Xiaobing Zuo,,${ }^{\S}$ Gavin Touponse, ${ }^{\dagger}$ Thorsten B. Blum, ${ }^{\ddagger}$ Khalid Salaita, ${ }^{\dagger}$ Jan Pieter Abrahams, ${ }^{\star},{ }^{\text {II }}$ and Vincent P. Conticello*†

$\dagger^{\dagger}$ Department of Chemistry, Emory University, Atlanta, Georgia 30322, United States

\$ Paul Scherrer Institut, CH-5232 Villigen Switzerland

${ }^{\S}$ X-ray Science Division, Argonne National Laboratory, Lemont, Illinois 60439, United States

II Center for Cellular Imaging and NanoAnalytics, Biozentrum, University of Basel, CH-4058, Basel, Switzerland 


\section{Supporting Information}
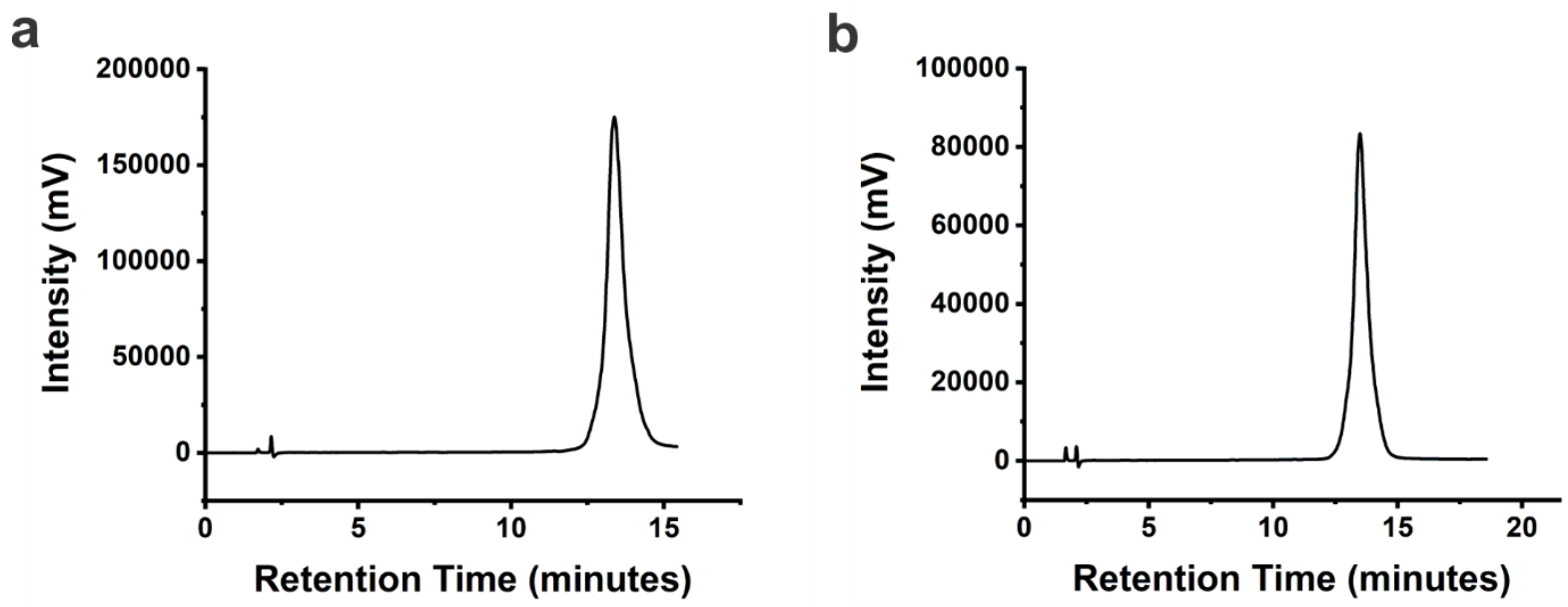

Figure S1. HPLC traces of (a) $\mathbf{4 S}(X)_{444}$ and (b) $\mathbf{4 R}(X)_{444}$.

a

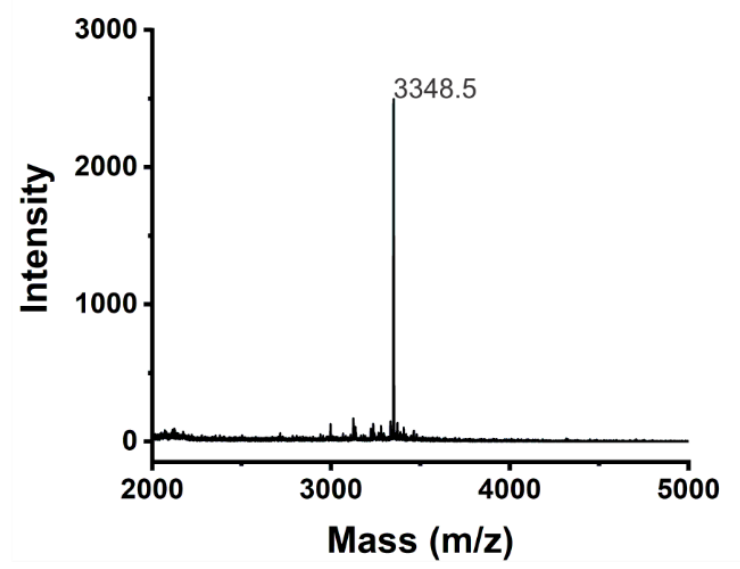

b

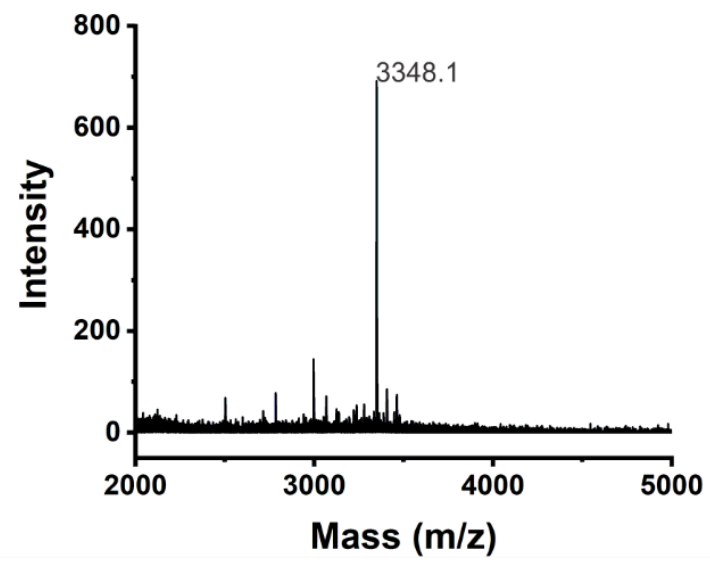

Figure S2. MALDI-TOF mass spectra of purified (a) $\mathbf{4 S}(\mathbf{X})_{444}, \mathrm{~m} / \mathrm{z}=3348.5\left(\mathrm{M}+\mathrm{H}^{+}\right)$and (b) $4 \mathbf{R}(\mathbf{X})_{444,} \mathrm{~m} / \mathrm{z}=3348.1\left(\mathrm{M}+\mathrm{H}^{+}\right)$. 

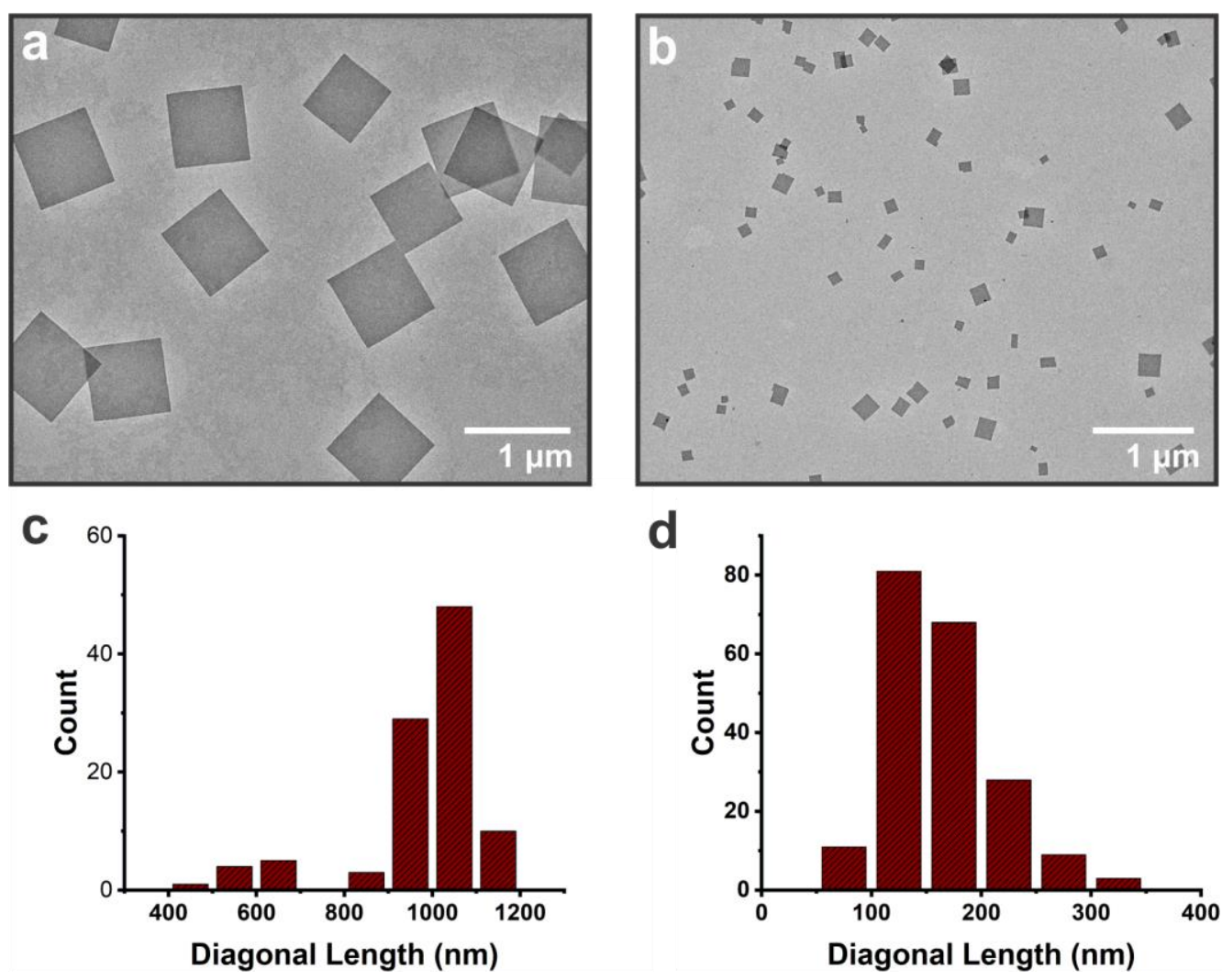

Figure S3. Stained TEM images of (a) $\mathbf{4 S}(X)_{444}(2 \mathrm{mg} / \mathrm{mL})$ and (b) $\mathbf{4 R}(\mathbf{X})_{444}(1 \mathrm{mg} / \mathrm{mL})$ nanosheets. Nanosheets were prepared using the thermal annealing process (see experimental section). Images were collected after 2 weeks of assembly time at $4^{\circ} \mathrm{C}$ and room temperature for $4 S(X)_{444}$ and $4 R(X)_{444}$ nanosheets, respectively. Corresponding diagonal length distributions of (c) $4 \mathbf{S}(\mathbf{X})_{444}$ nanosheets $(2 \mathrm{mg} / \mathrm{mL})$ after 2 weeks of incubation at $4^{\circ} \mathrm{C}: 977 \pm 146 \mathrm{~nm}$ (based on 100 counts) and (d) $\mathbf{4 R}(\mathbf{X})_{444}$ nanosheets $(1 \mathrm{mg} / \mathrm{mL})$ after 2 weeks of incubation at room temperature: $163 \pm 49 \mathrm{~nm}$ (based on 200 counts). 

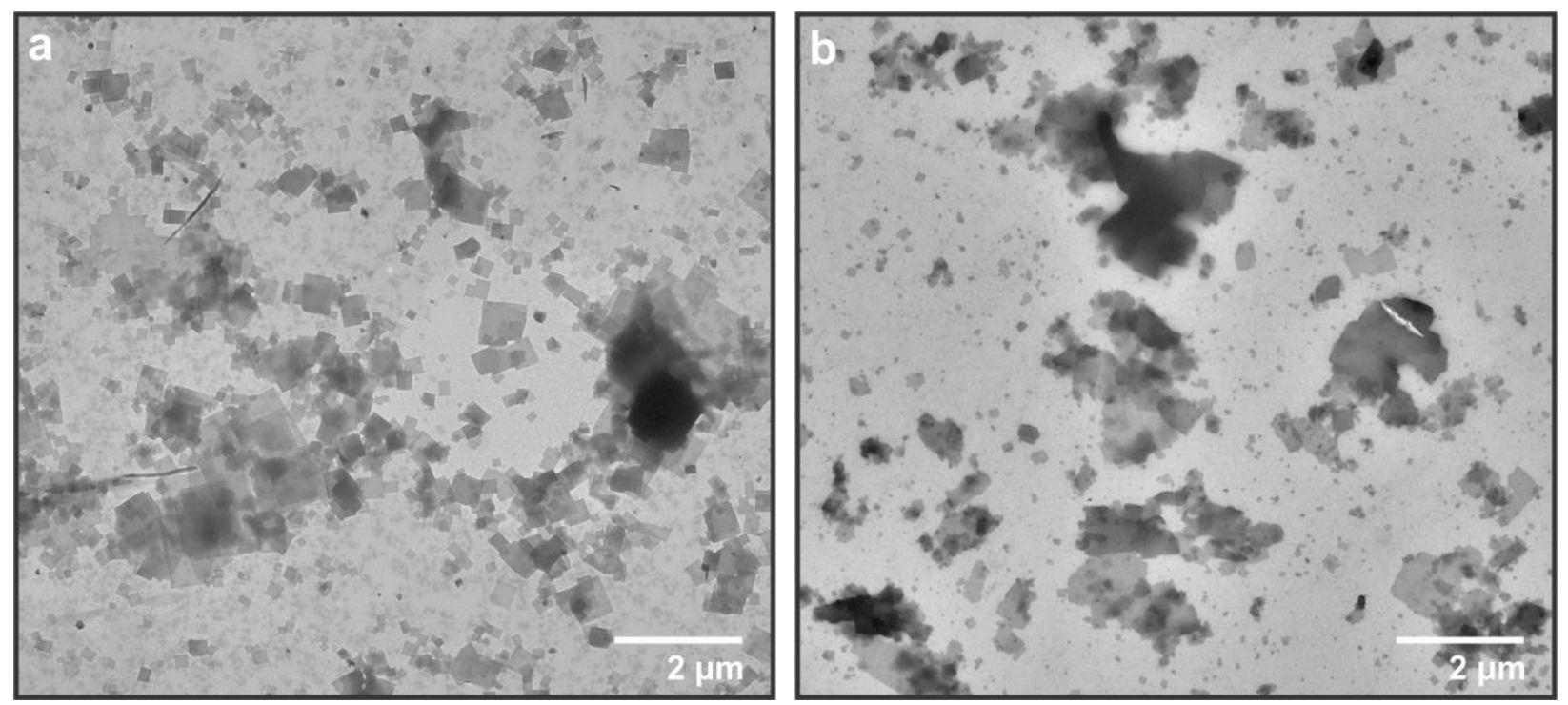

Figure S4. Stained TEM images of (a) $\mathbf{4 S}(\mathbf{X})_{\mathbf{4 4 4}}(2 \mathrm{mg} / \mathrm{mL})$ and (b) $\mathbf{4 R}(\mathbf{X})_{\mathbf{4 4 4}}(1 \mathrm{mg} / \mathrm{mL})$ assembled at $4^{\circ} \mathrm{C}$ and room temperature, respectively, after 2 weeks of assembly time. No thermal annealing protocol was used. Ill-defined, polymorphic, and aggregated structures were observed indicating the importance of thermal input for producing homogeneous distributions of well-defined nanosheets.
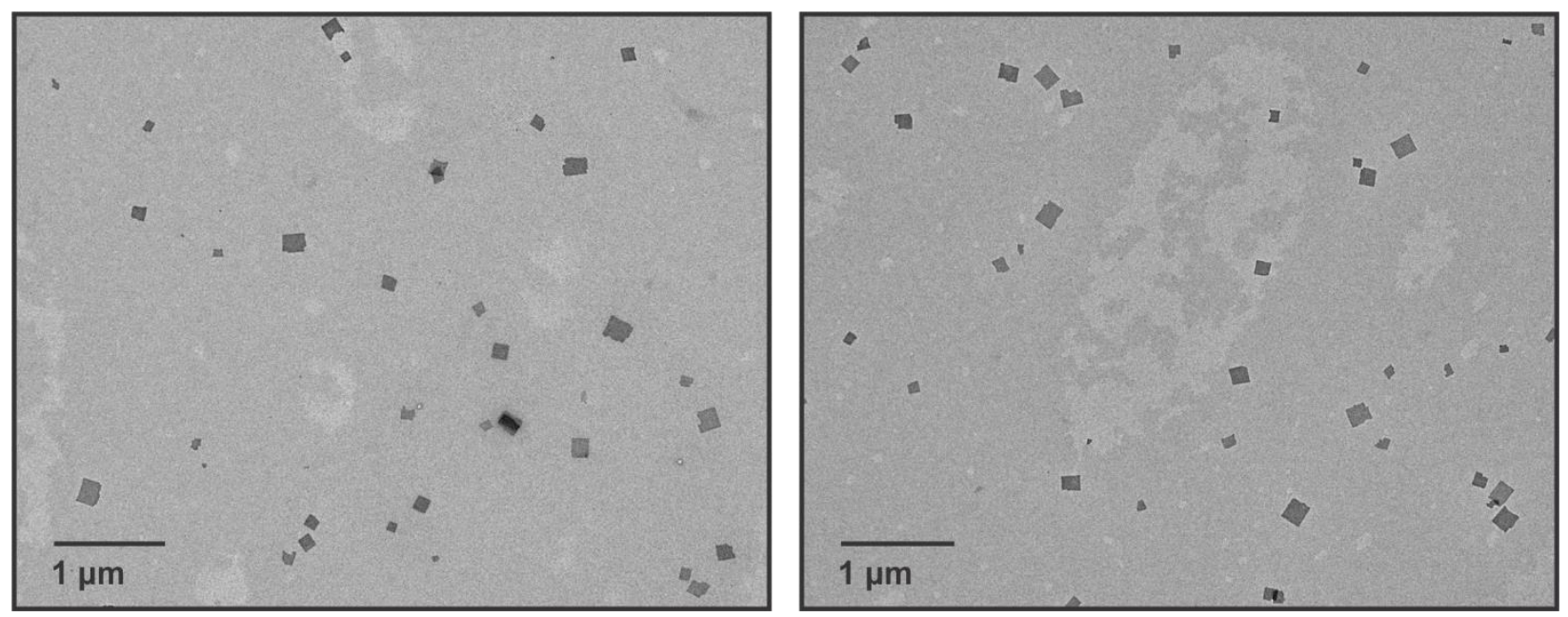

Figure S5. Stained TEM images of $\mathbf{4 R}(\mathbf{X})_{444}$ nanosheets annealed at $40^{\circ} \mathrm{C}$ in the presence of $\mathbf{4 S}(X)_{444}$ peptide immediately before cooling. 

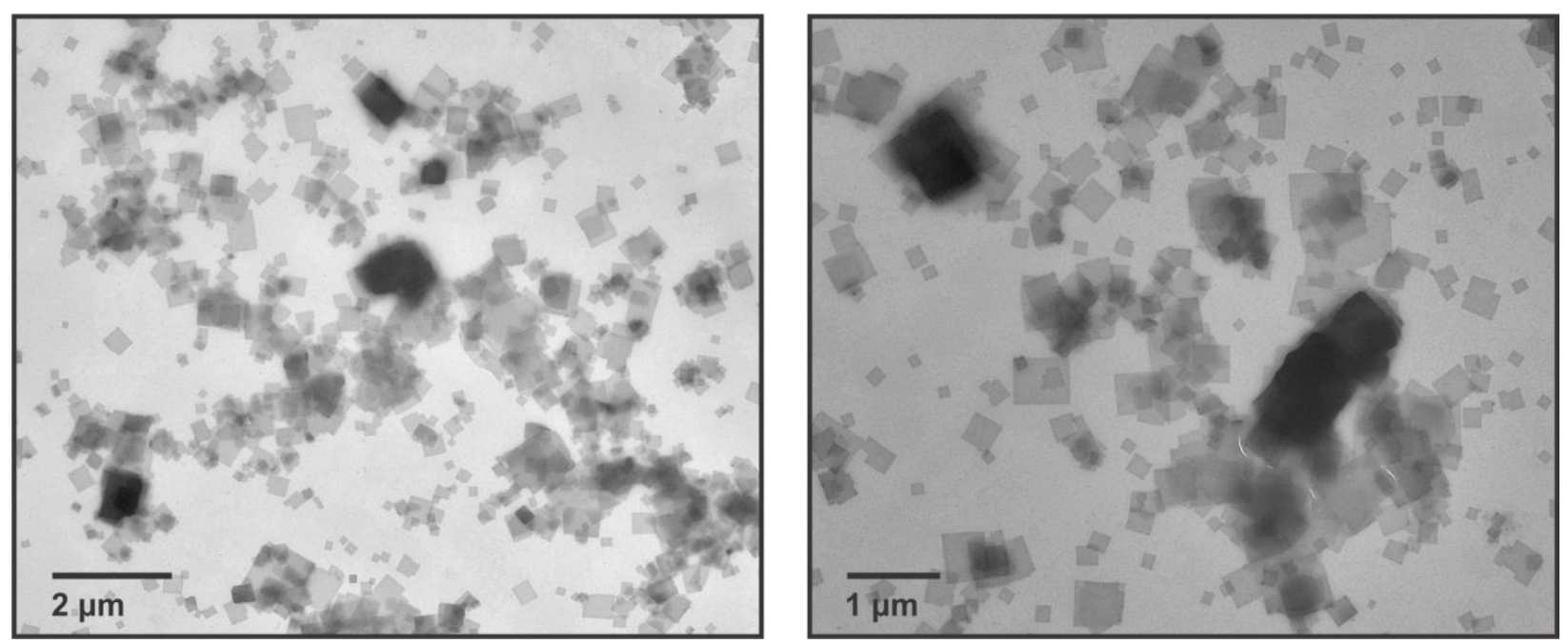

Figure S6. Stained TEM images of seeded growth assembly experiments conducted in the absence of thermal annealing. A solution of $\mathbf{4 R}(\mathbf{X})_{\mathbf{4 4 4}}$ nanosheets $(0.2 \mathrm{mg} / \mathrm{mL})$ was added to $\mathbf{4 S}(\mathbf{X})_{\mathbf{4 4 4}}(2$ $\mathrm{mg} / \mathrm{mL}$ ), and the mixture was incubated at $4{ }^{\circ} \mathrm{C}$ for 11 days. Polymorphic and ill-defined assemblies were observed. 
a

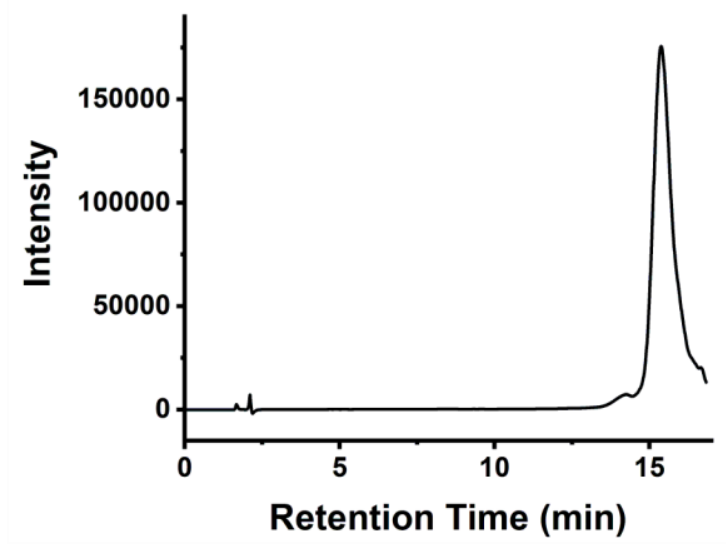

C

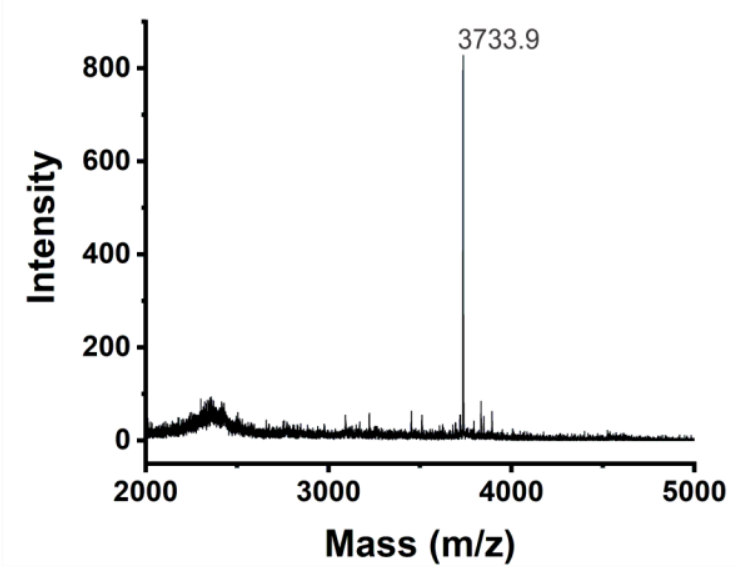

b

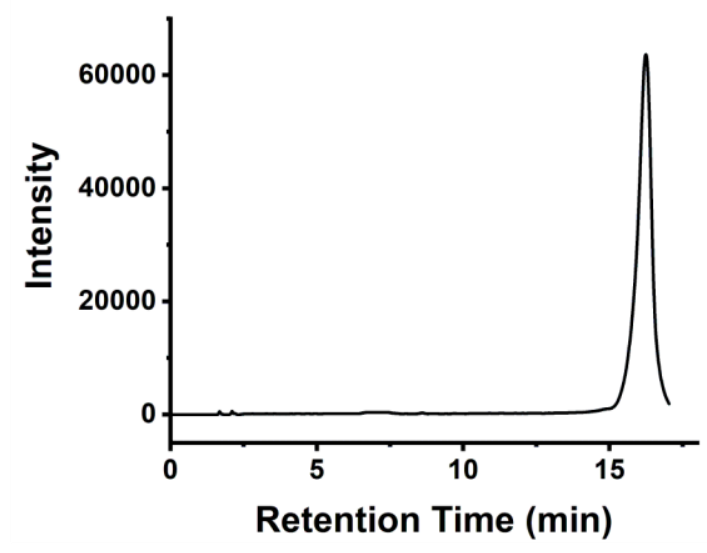

d

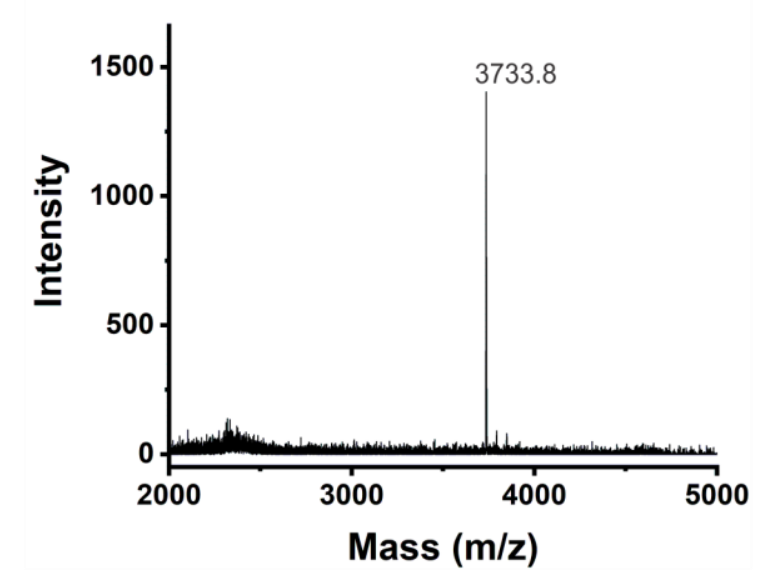

Figure S7. HPLC traces of (a) b-4R(X) 444 and (b) b-4S(X) 444 . MALDI-TOF spectra of (c) b$\mathbf{4 R}(\mathbf{X})_{444,} \mathrm{~m} / \mathrm{z}=3733.9\left(\mathrm{M}+\mathrm{H}^{+}\right)$and $(\mathrm{d}) \mathbf{b}-\mathbf{4 S}(\mathbf{X})_{444}, \mathrm{~m} / \mathrm{z}=3733.8\left(\mathrm{M}+\mathrm{H}^{+}\right)$. 

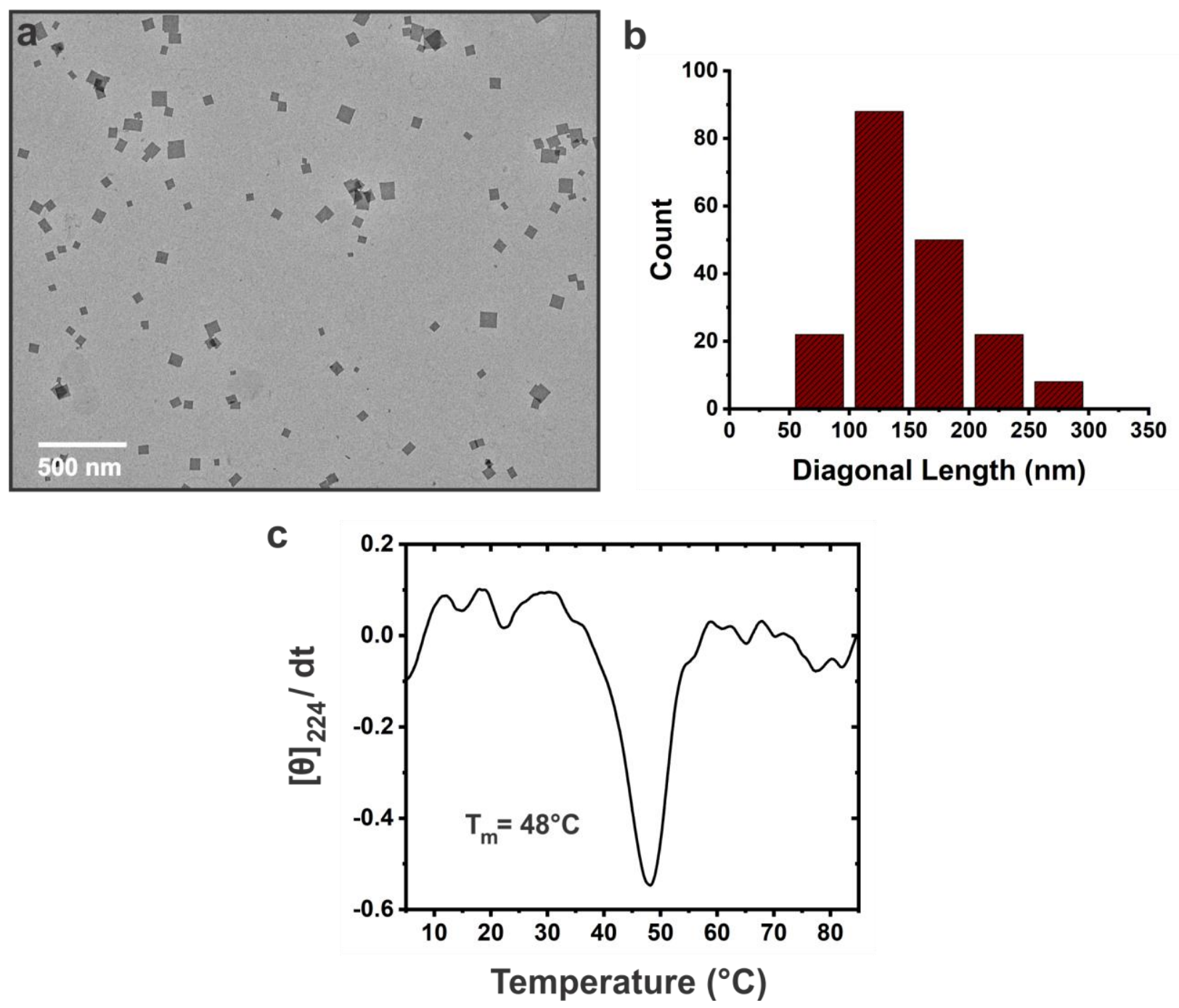

Figure S8. (a) Stained TEM image of $\mathbf{b}-\mathbf{4 R}(\mathbf{X}) \mathbf{4 4 4}$ nanosheets ( $5 \%$ biotin-doped; $1 \mathrm{mg} / \mathrm{mL}$ ) after 2 weeks of assembly at room temperature. (b) Diagonal length distribution of $\mathbf{b}-\mathbf{4 R}(\mathbf{X})_{\mathbf{4 4 4}}$ nanosheets: $149 \pm 47 \mathrm{~nm}$ (based on 190 counts). (c) First derivative of the CD signal of $\mathbf{b}-\mathbf{4 R}(\mathbf{X})_{444}$ nanosheets $(0.2 \mathrm{mg} / \mathrm{mL})$ at $224 \mathrm{~nm}$ as a function of temperature. $\mathrm{T}_{\mathrm{m}}=48^{\circ} \mathrm{C}$. 

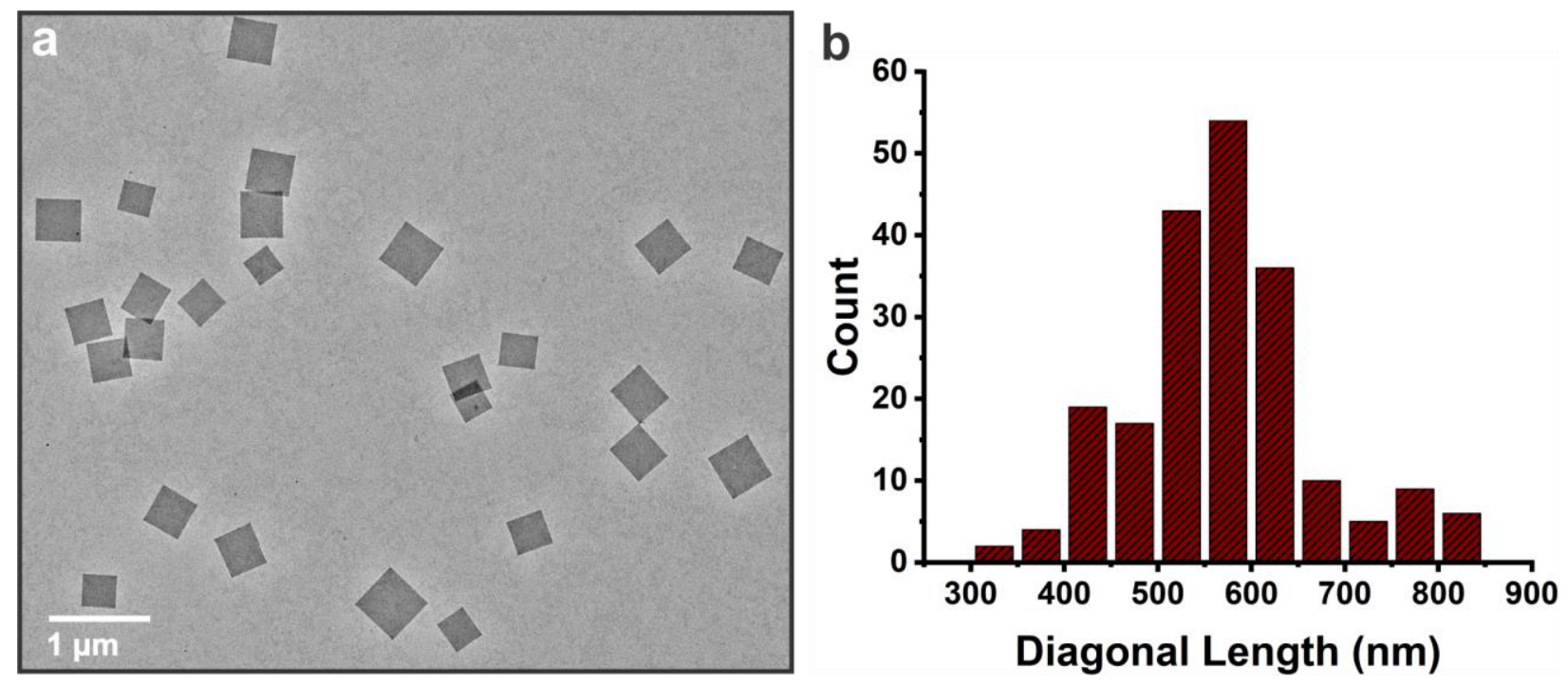

Figure S9. (a) Stained TEM image of b-4R(X)444@4S(X)444 after 2 weeks of assembly time at $4^{\circ} \mathrm{C}$. (b) Diagonal length distribution of $\mathbf{b}-\mathbf{4 R}(X)_{444} @ \mathbf{4 S}(X)_{444}$ nanosheets: $562 \pm 87 \mathrm{~nm}$ (based on 200 counts).
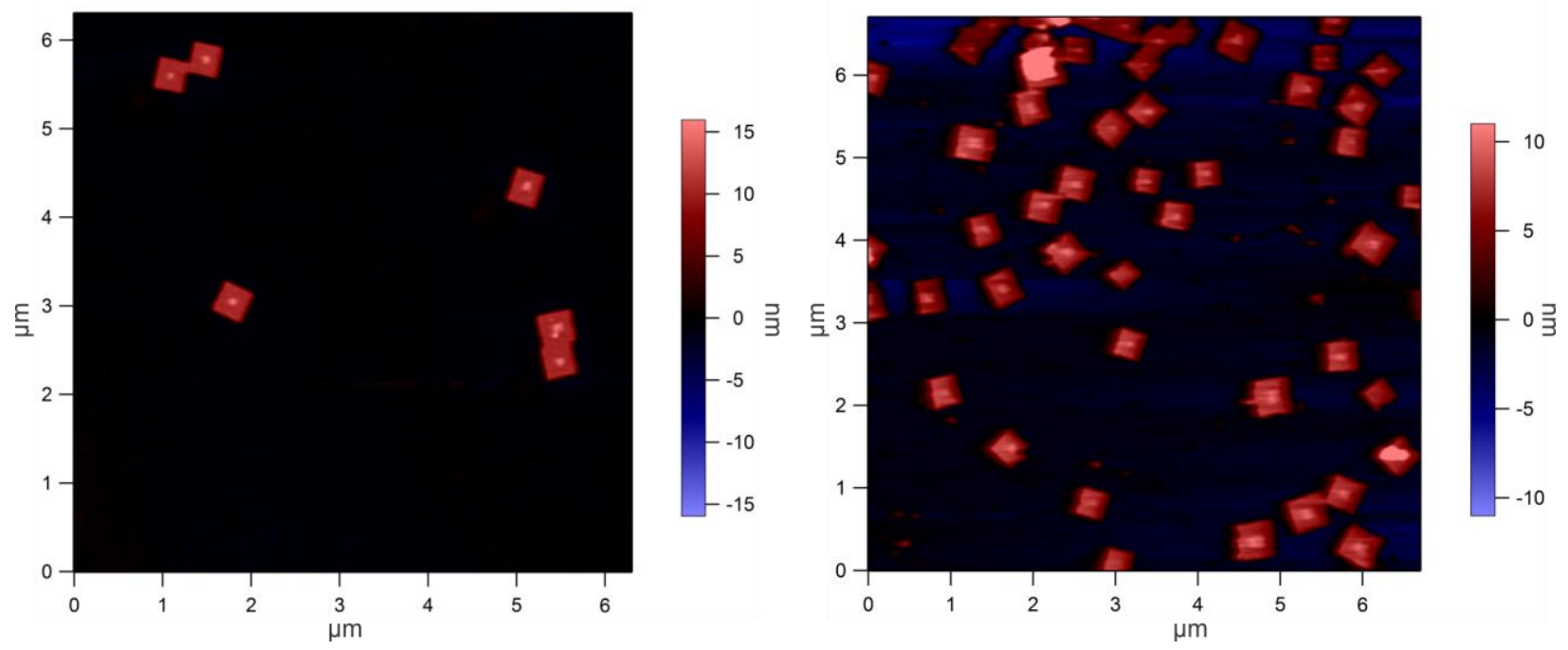

Figure S10. AFM height images of streptavidin-coated b-4R(X)444@ $\mathbf{4 S}(X)_{\mathbf{4 4 4}}$ nanosheets. 

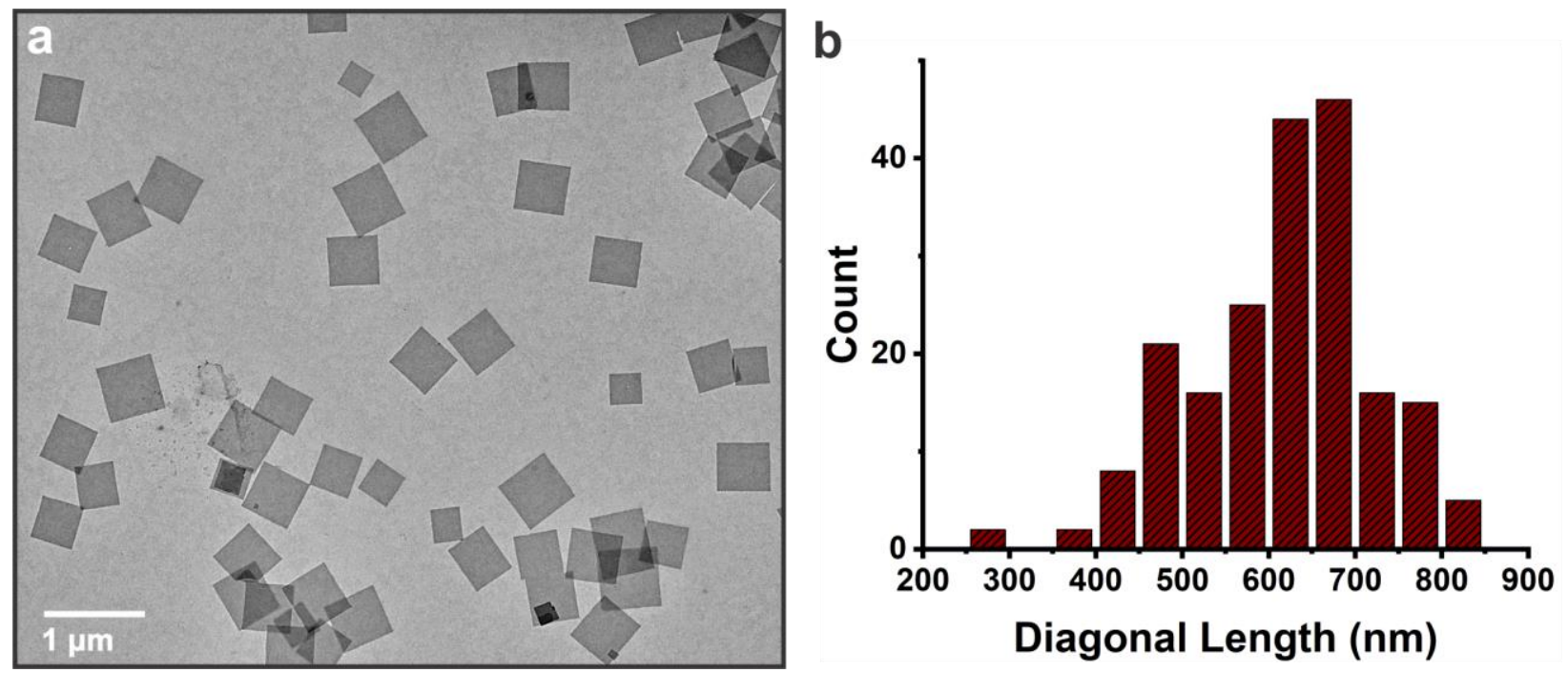

Figure S11. (a) Stained TEM image of $\mathbf{4 R}(X) \mathbf{4 4 4} @ \mathbf{b}-\mathbf{4 S}(X) 444$ after 2 weeks of assembly time. (b) Diagonal length distribution of $\mathbf{4 R}(\mathbf{X})_{\mathbf{4 4 4}} @ \mathbf{b}-\mathbf{4 S}(\mathbf{X})_{\mathbf{4 4 4}}$ nanosheets: $616 \pm 104 \mathrm{~nm}$ (based on 200 counts).
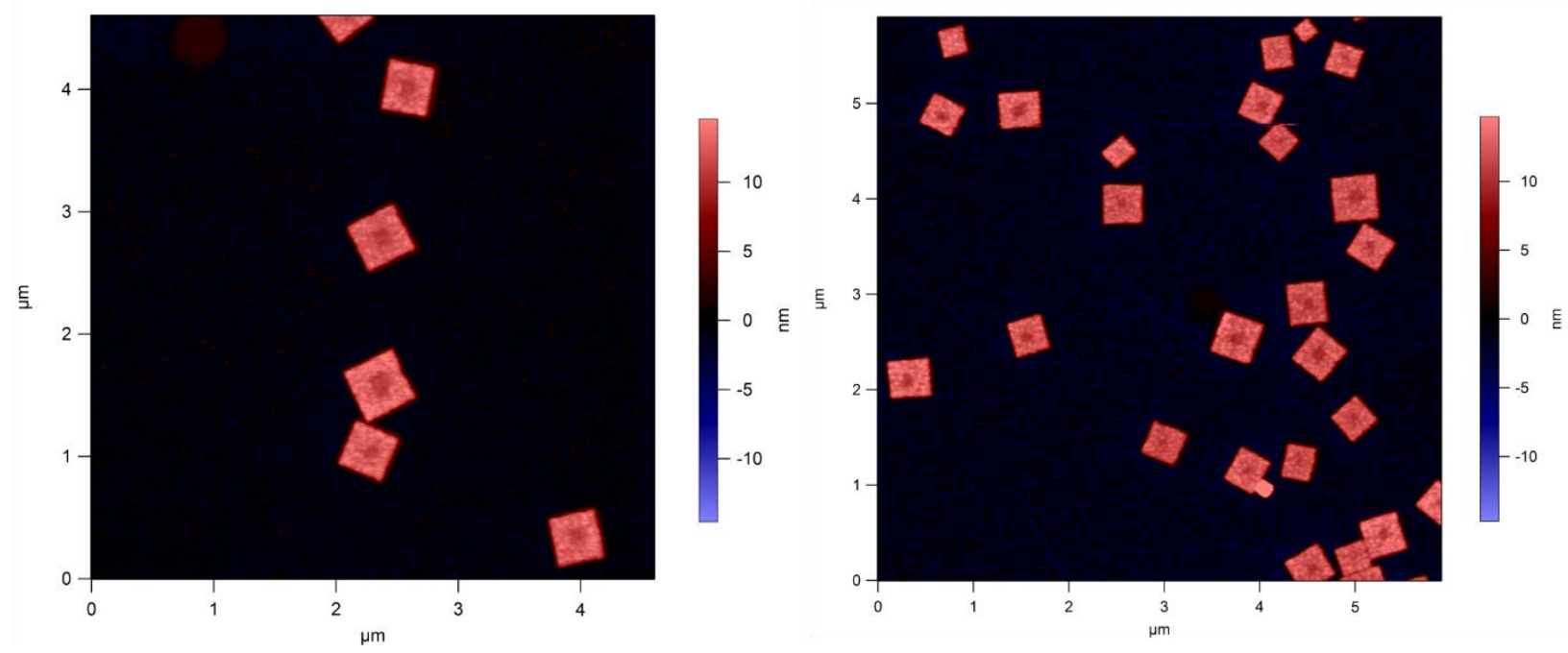

Figure S12. AFM height images of streptavidin-coated $4 R(X)_{444} @ b-4 S(X)_{444}$ nanosheets. 


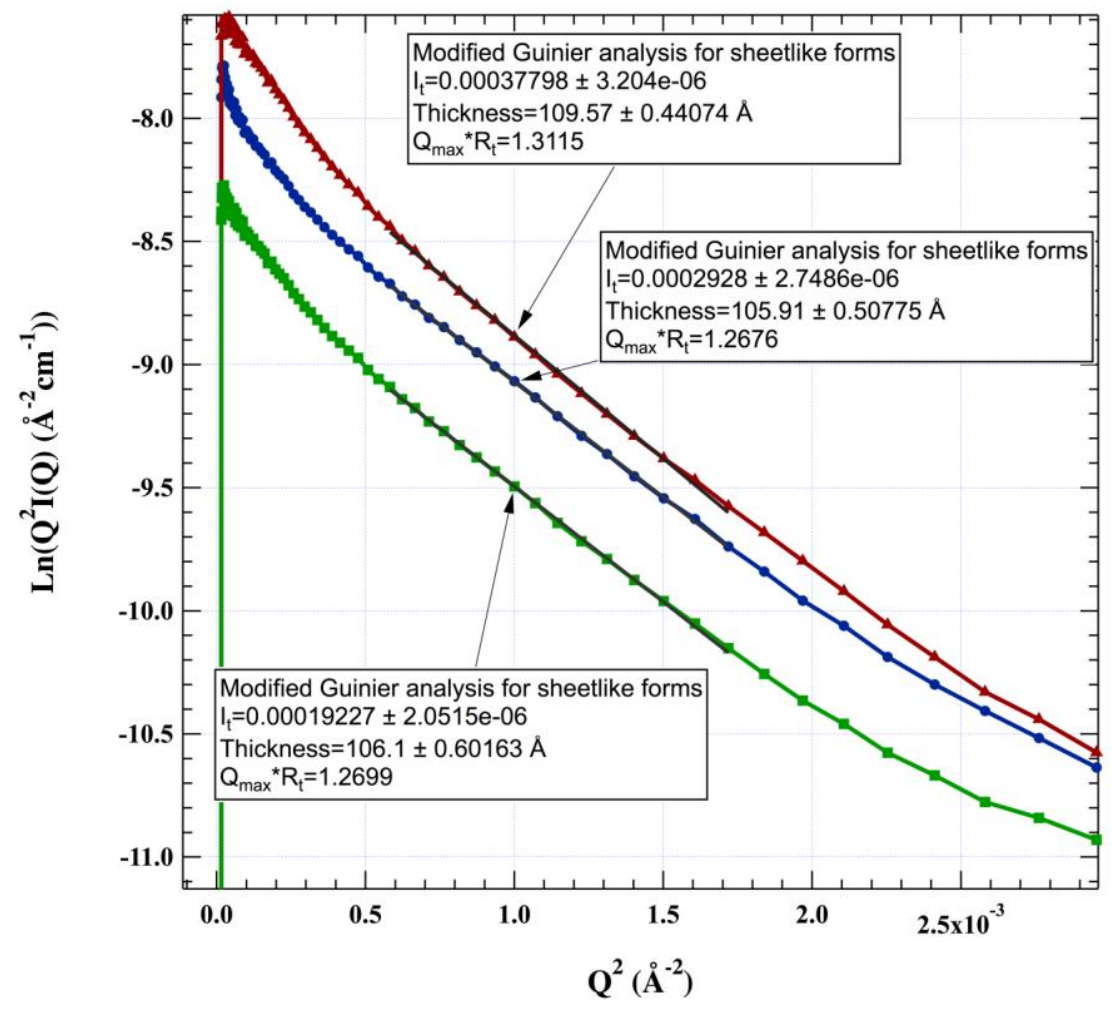

Figure S13. Guinier plots of $\mathbf{4 S}(X) \mathbf{4 4 4}$ (blue line), $\mathbf{4 R}(X) \mathbf{4 4 4}$ (red line), and $\mathbf{4 R}(X)_{\mathbf{4 4 4}} @ \mathbf{4 S}(X) \mathbf{4 4 4}$ (green line) nanosheets in $20 \mathrm{mM}$ MOPS buffer ( $\mathrm{pH}$ 7.0) with fit for sheet-like form.

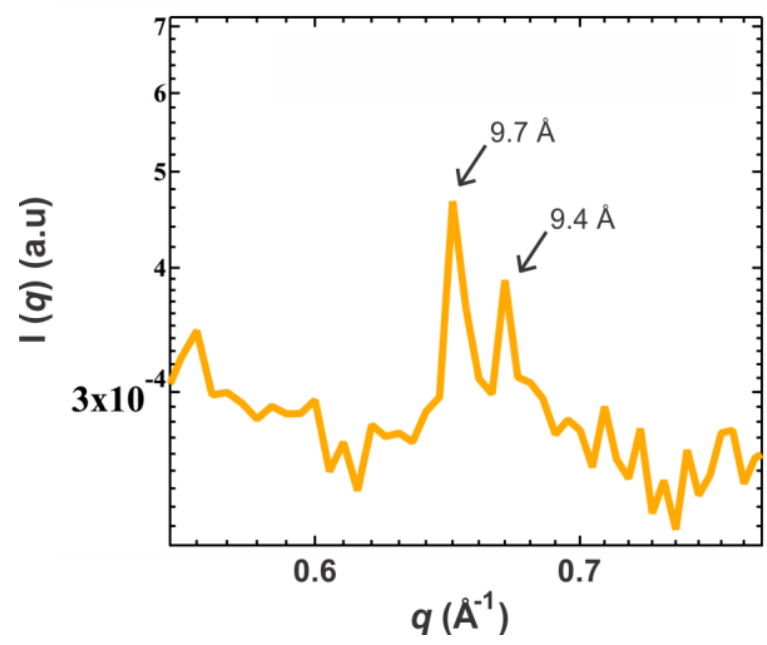

Figure S14. Zoomed-in image of the synchrotron scattering profile of $\mathbf{4 R}(X) \mathbf{4 4 4}$ ( $q$ values between $\sim 0.6$ and $\sim 0.7 \AA^{-1}$ ) revealing the presence of two Bragg peaks corresponding to $d$-spacing of $9.7 \AA$ and $9.4 \AA$. 


\begin{tabular}{|l|c|c|c|c|c}
\hline & Single-block Nanosheet & Diblock Nanosheet & Multiple crystals & Uncertain & Total \\
\hline Images & 60 & 403 & 75 & 36 & 574 \\
\hline Percent & $\mathbf{1 3 . 0 \%}$ & $\mathbf{8 7 . 0 \%}$ & & & \\
\hline
\end{tabular}

Figure S15. Analysis of nanocrystals collected from cryo-TEM. "Single-block Nanosheet" refers to single-component nanosheets (self-nucleated $\mathbf{4 S}(\mathrm{X})_{\mathbf{4 4 4}}$ sheets). "Diblock Nanosheet" refers to 4R(X)444@4S $(X) 444$ nanosheets. "Multiple crystals" refers to the presence of many crystals or overlapping crystals present in the image. "Uncertain" refers to images where only a small portion of a crystal is observed. Product distribution of $13.0 \%$ and $87.0 \%$ for single-block and diblock assemblies, respectively, were calculated from a total of 463 nanocrystals. Crystals observed in images designated "multiple crystals" and "uncertain" were not used in the calculation because we are unable to determine if the sheets were either single-block or diblock assemblies. 


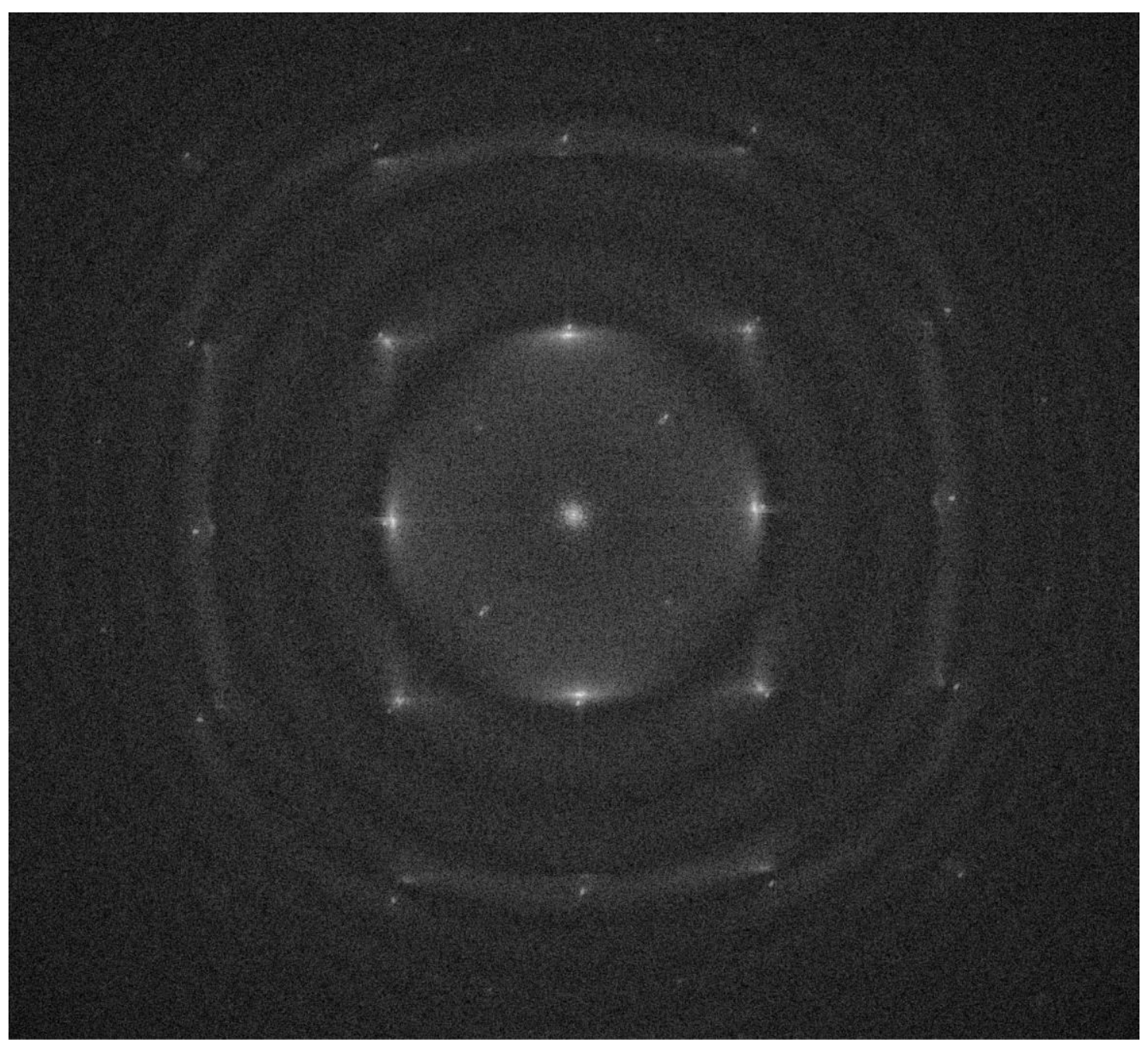

Figure S16. FFT of a movie-corrected cryo-TEM image of the $\mathbf{4 R}(X)_{444} @ \mathbf{4 S}(X)_{444}$ nanosheet shown in Figure 3a-c. Two sets of Bragg spots are observed indicating the presence of two distinct crystal lattices. 


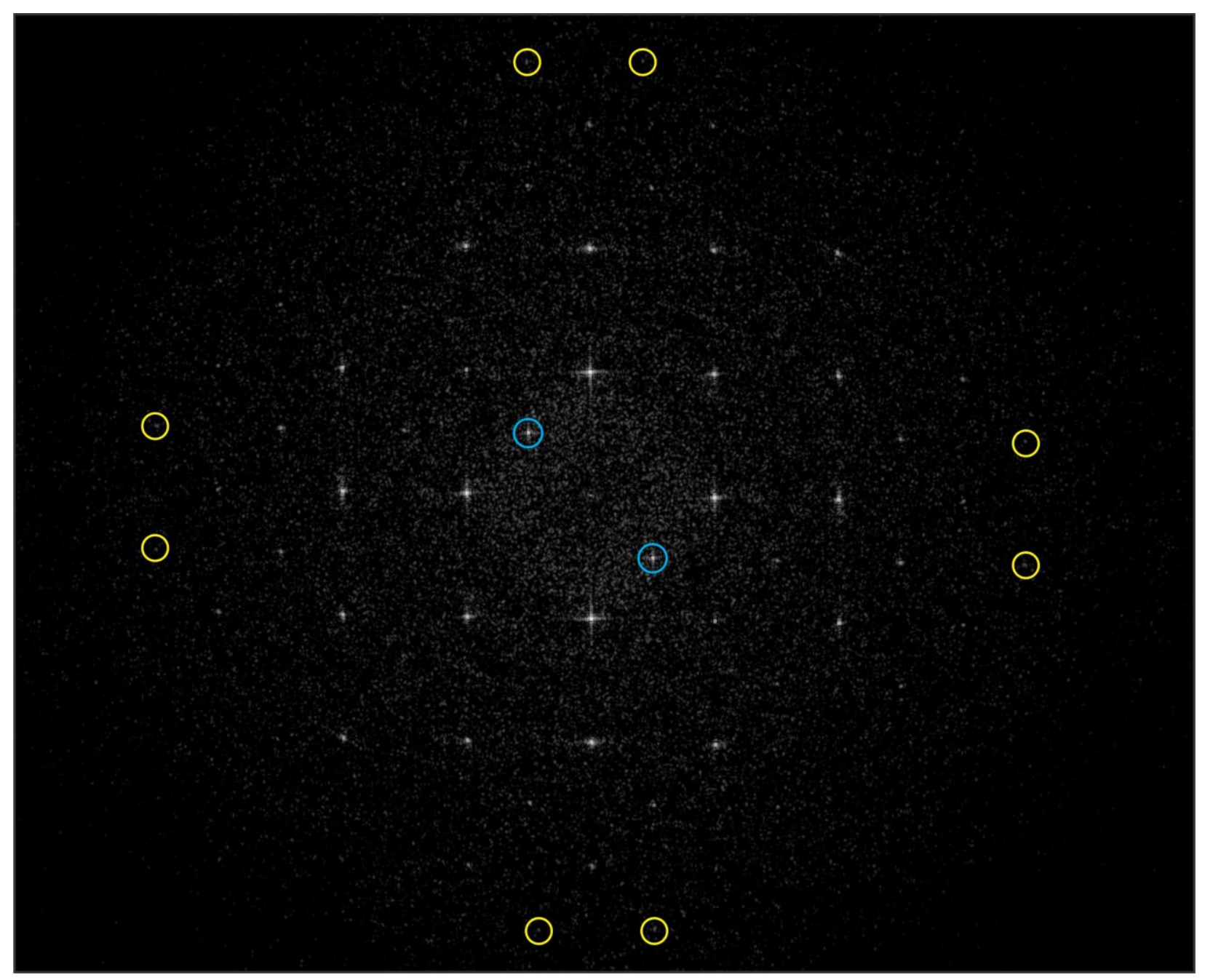

Figure S17. Selected area FFT of the $\mathbf{4 R}(X)_{444}$ core with selected Bragg spots circled. Yellow circles: $d$-spacing $=3.86 \AA$. Blue circles: $d$-spacing $=\sim 19.1 \AA$. 
Figure S18. Selected area FFT of the $\mathbf{4 S}(X)_{444}$ shell with selected Bragg spots circled. Yellow circles: $d$-spacing $=6.4 \AA$. Blue circles: $d$-spacing $=20.0-20.2 \AA$. 

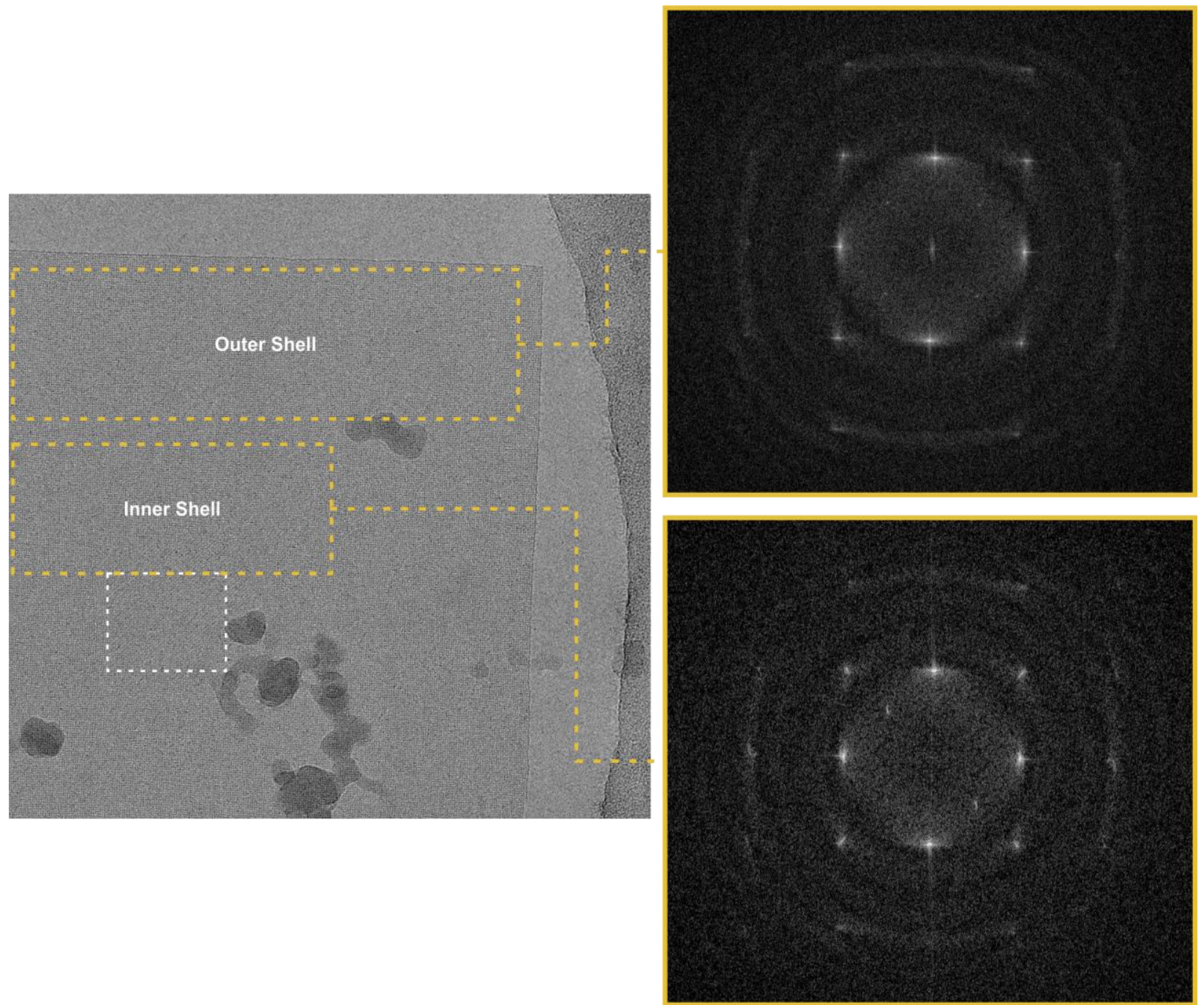

Figure S19. FFTs of different regions within the shell sector of the $4 R(X)_{444} @ 4 S(X)_{444}$ nanosheet. White dashed box indicates the location of the core sector. 


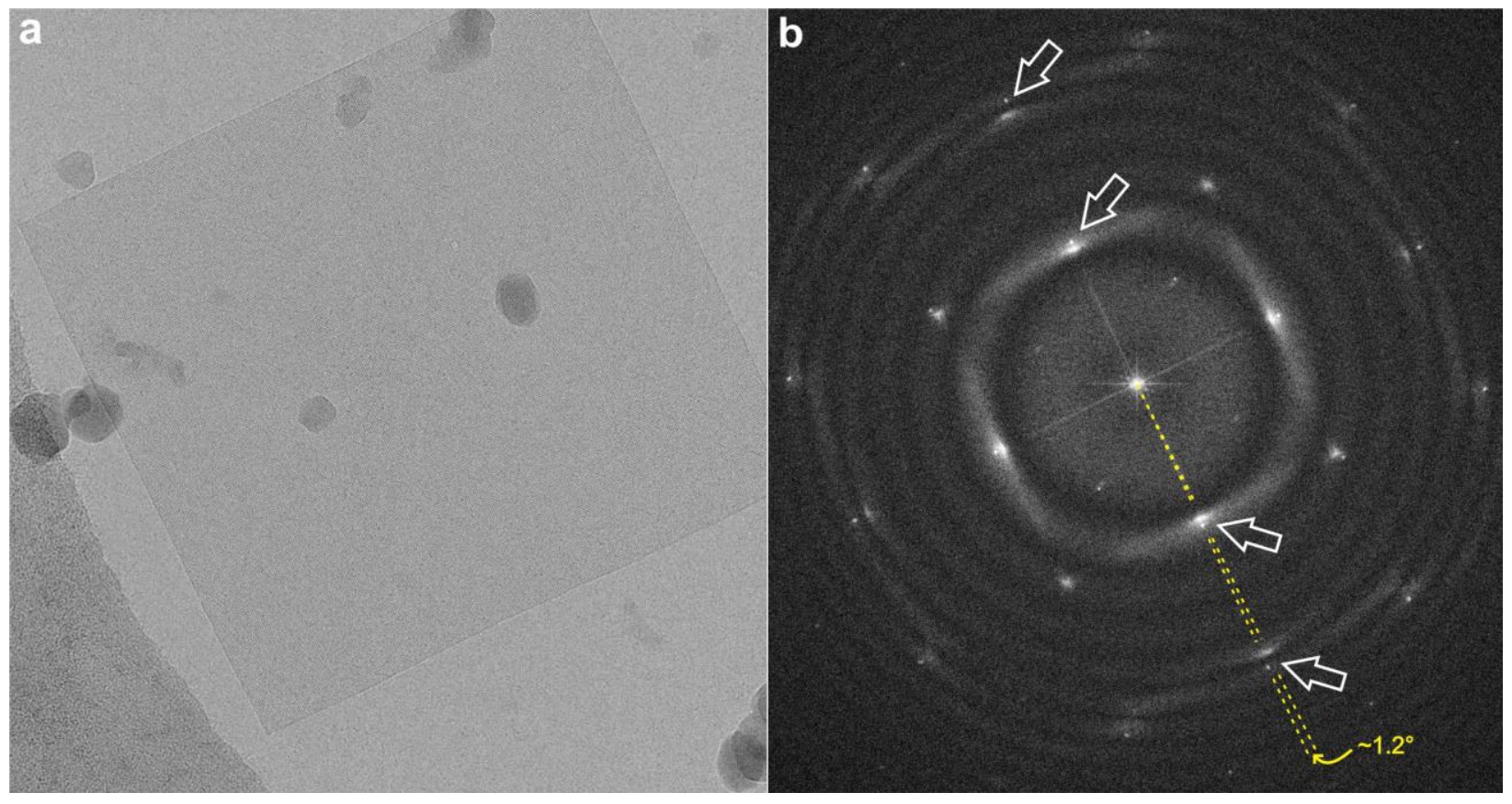

Figure S20. (a) Cryo-TEM image of a $\mathbf{4 R}(X)_{\mathbf{4 4 4}} @ \mathbf{4 S}(X)_{\mathbf{4 4 4}}$ nanosheet. (b) FFT analysis reveals a slight discrepancy in lattice orientation between the core and shell lattice. White arrows show the slight angular offset $\left(\sim 1.2^{\circ}\right)$ between Bragg spots corresponding to both lattices. 


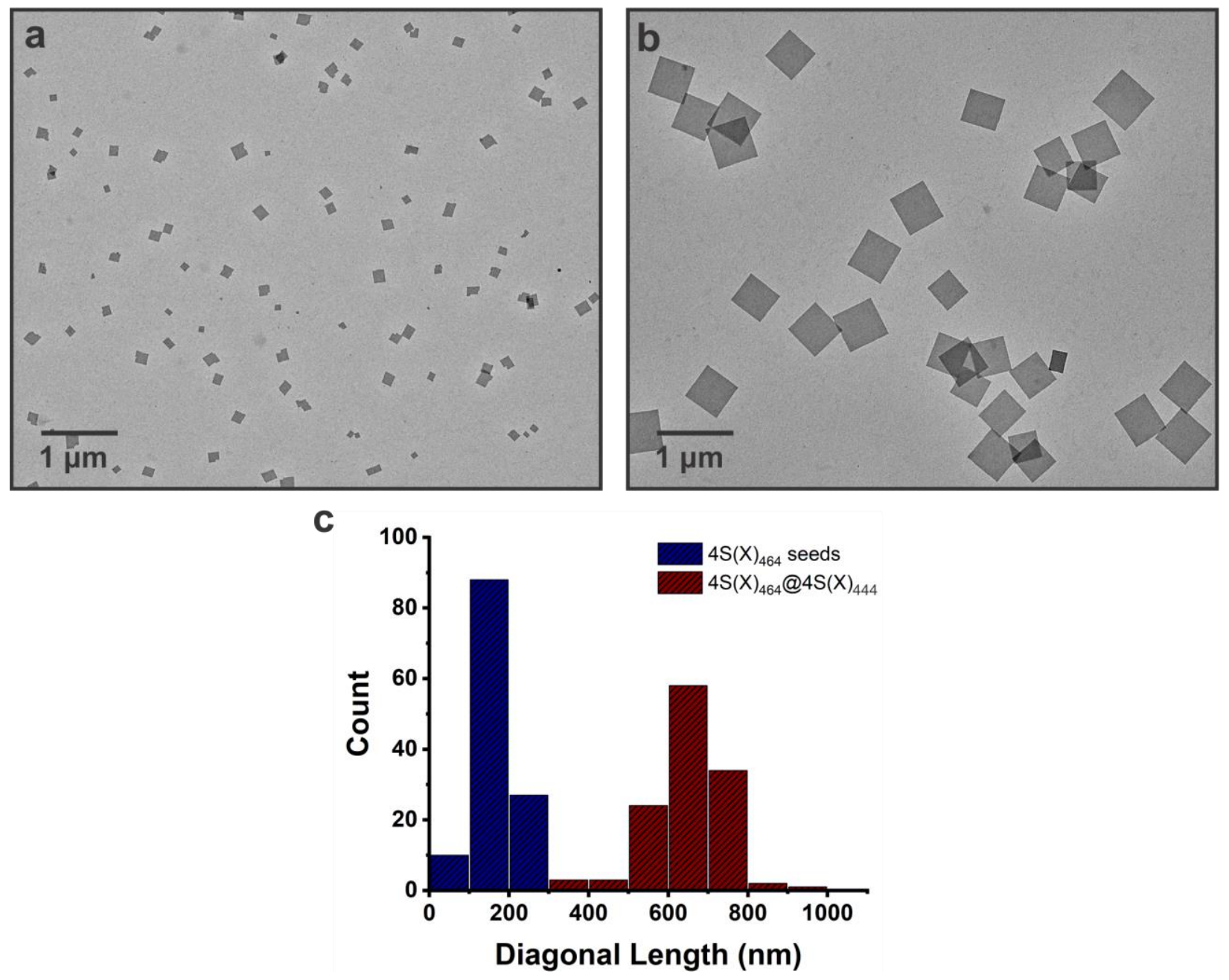

Figure S21. Stained TEM images of (a) 4S(X)464 nanosheet seeds and (b) $4 \mathrm{~S}(X) \mathbf{4 6 4} @ \mathbf{4 S}(X) \mathbf{4 4 4}$ nanosheet after 2 weeks of assembly time at room temperature and $4{ }^{\circ} \mathrm{C}$, respectively. (c) Diagonal length distribution of $\mathbf{4 S}(\mathbf{X})_{464}$ nanosheet seeds (blue): $163 \pm 42 \mathrm{~nm}$, based on 125 counts and 4S $(X)_{464} @ \mathbf{4 S}(X)_{444}$ nanosheets (red) after two weeks of assembly time: $649 \pm 95 \mathrm{~nm}$, based on 125 counts. 


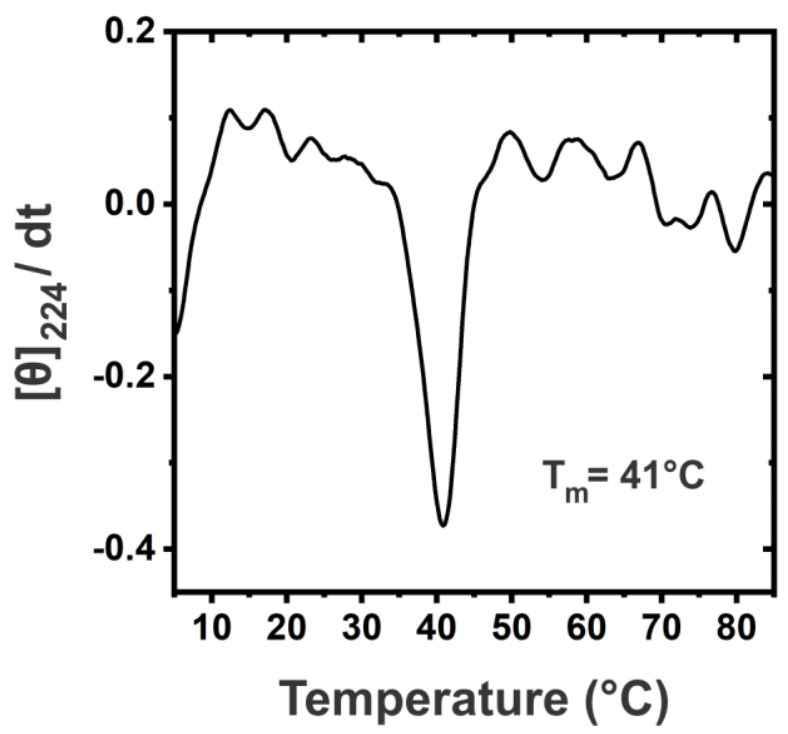

Figure S22. First derivative of the CD signal of $\mathbf{4 S}(\mathbf{X})_{464}$ nanosheets $(0.2 \mathrm{mg} / \mathrm{mL})$ at $224 \mathrm{~nm}$ as a function of temperature. $\mathrm{T}_{\mathrm{m}}=41^{\circ} \mathrm{C}$.

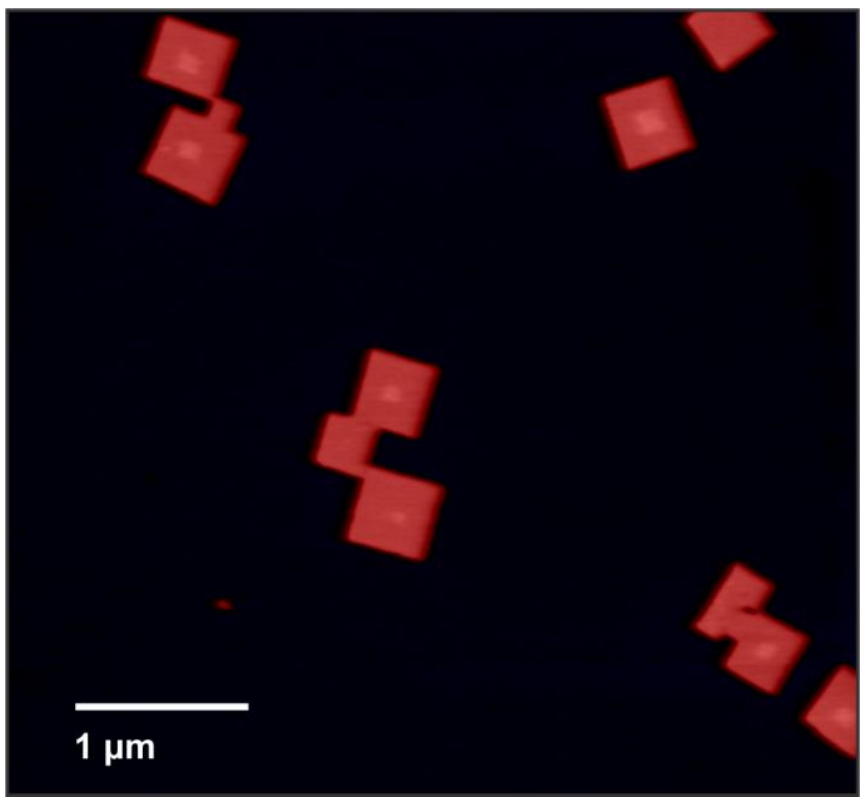

Figure S23. AFM height image of $4 S(X)_{464} @ 4 S(X)_{444}$ nanosheets. 


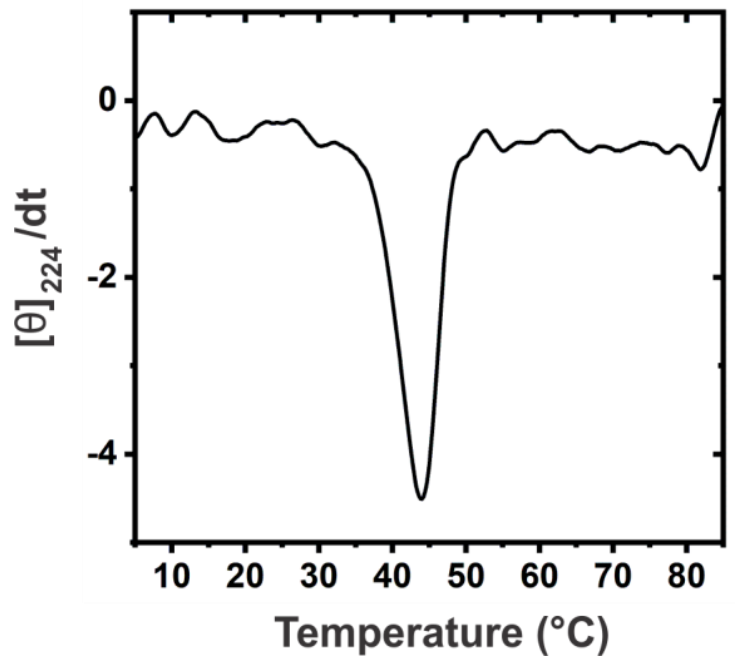

Figure S24. First derivative of the CD signal of $\mathbf{4 S}(\mathbf{X}) 454$ nanosheets $(2 \mathrm{mg} / \mathrm{mL})$ at $224 \mathrm{~nm}$ as a function of temperature. $\mathrm{T}_{\mathrm{m}}=44^{\circ} \mathrm{C}$.
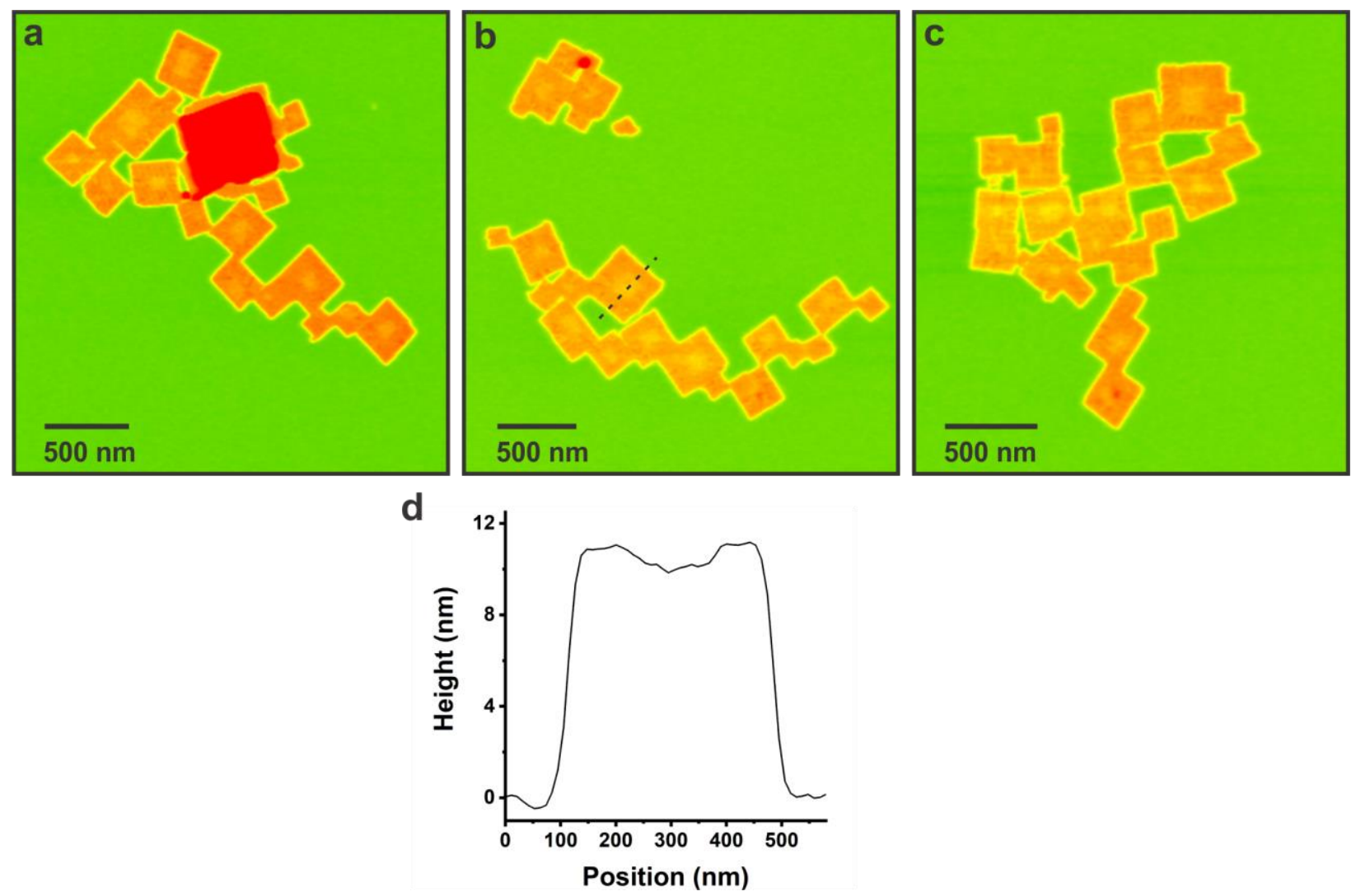

Figure S25. (a-c) AFM images of $\mathbf{4 R}(X)_{444} @ \mathbf{4 S}(X)_{454}$ nanosheets. A small number of selfnucleated $4 \mathrm{~S}(\mathrm{X}) 454$ nanosheets can also be observed. (d) AFM height trace of the dashed line shown in figure (b). 

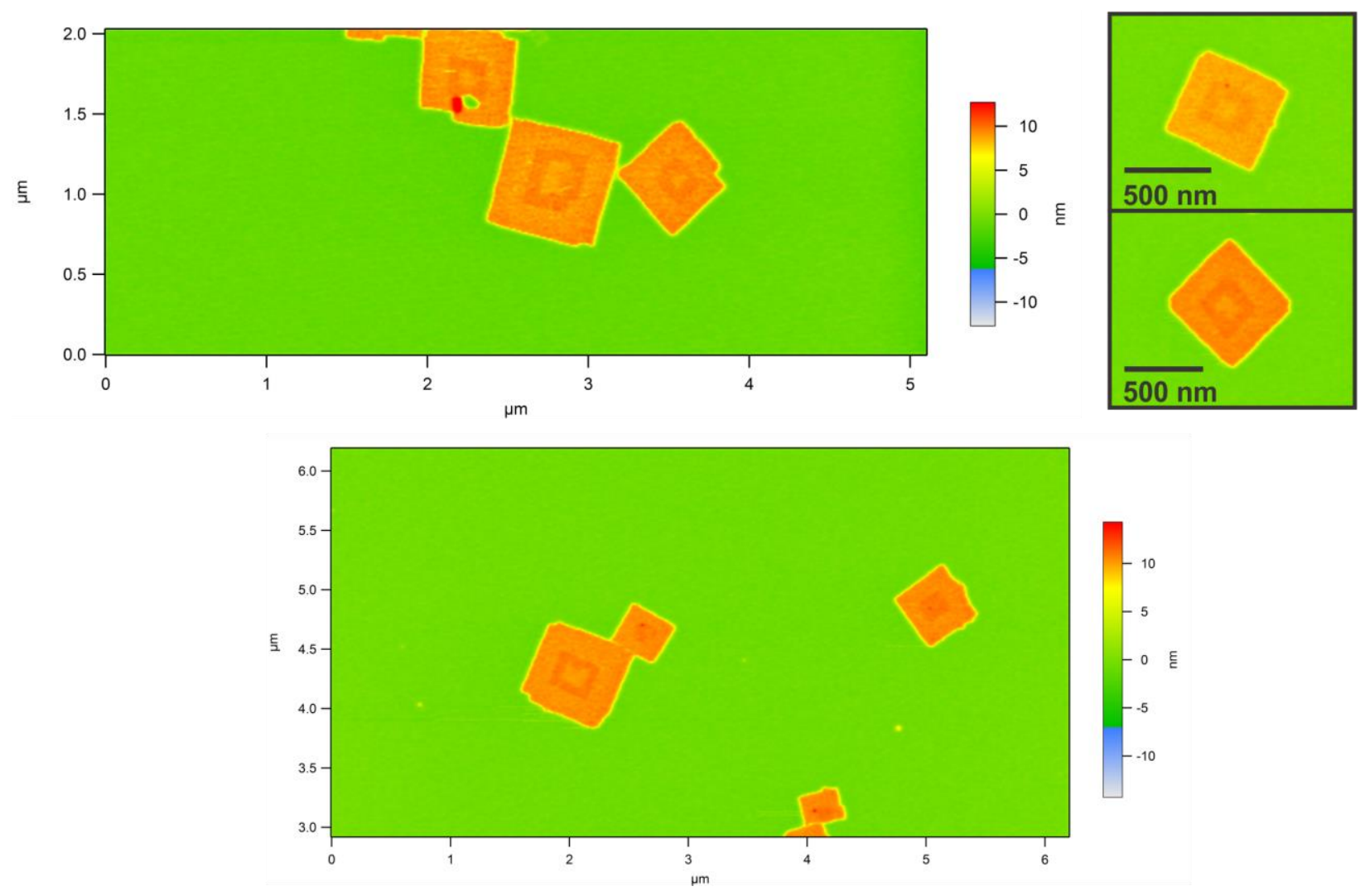

Figure S26. AFM images of 4R(X)444@4S(X)454@4S(X)444 nanosheets. Diblock nanosheets (4S $(X) 454 @ 4 S(X) 444)$ are also present. 


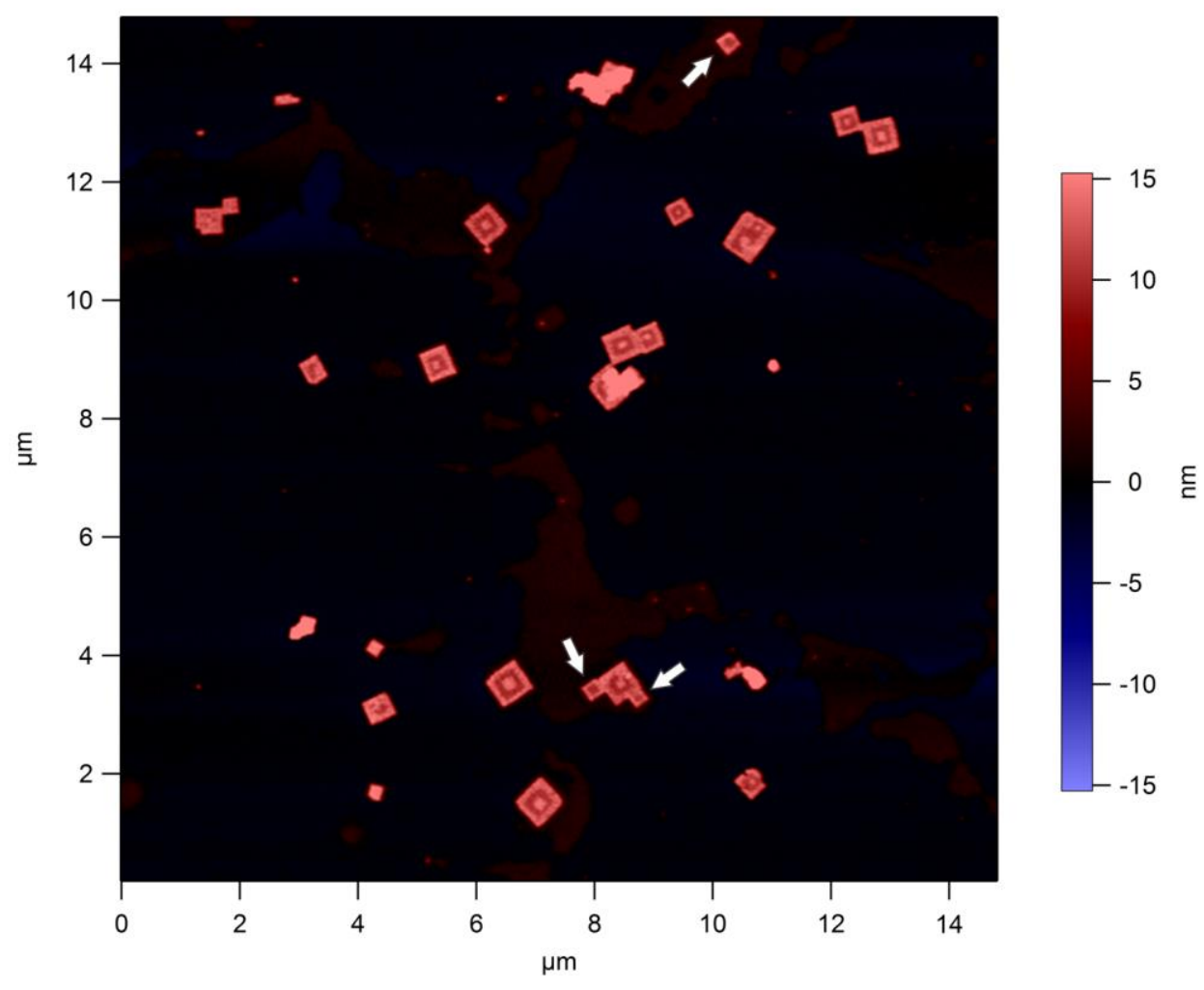

Figure S27. AFM image of b-4R(X)444@4S(X)454@b-4S $(X)_{444}$ nanosheets. White arrows indicate 4S $(X)_{454} @ b-4 S(X) 444$ diblock nanosheets (side product).
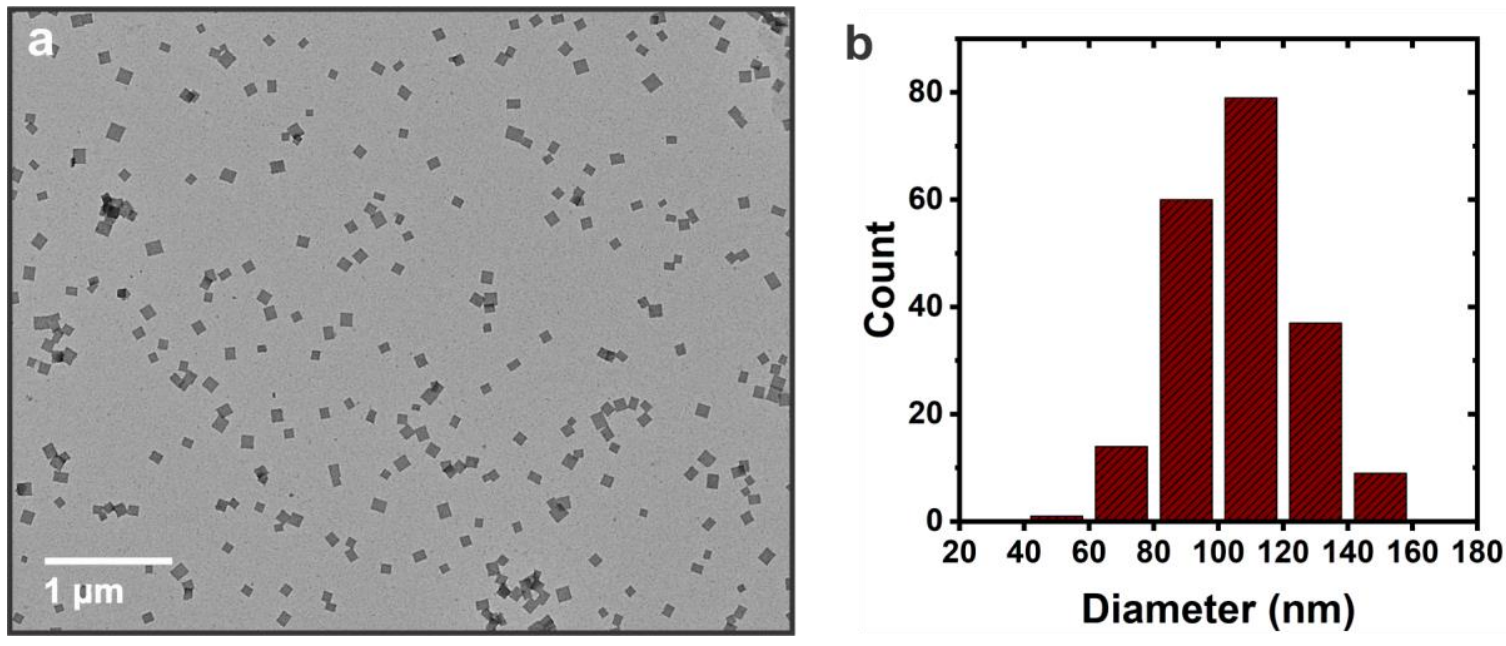

Figure S28. (a) Stained TEM image of $\mathbf{4 R}(\mathbf{X})_{444}$ nanosheets assembled in TAPS buffer ( $\mathrm{pH} \mathrm{8.0).}$ (b) Diagonal length distribution of $\mathbf{4 R}(\mathbf{X})_{\mathbf{4 4 4}}$ nanosheets assembled in TAPS buffer (pH 8.0): 106 $\pm 19 \mathrm{~nm}$ (based on 200 counts). 


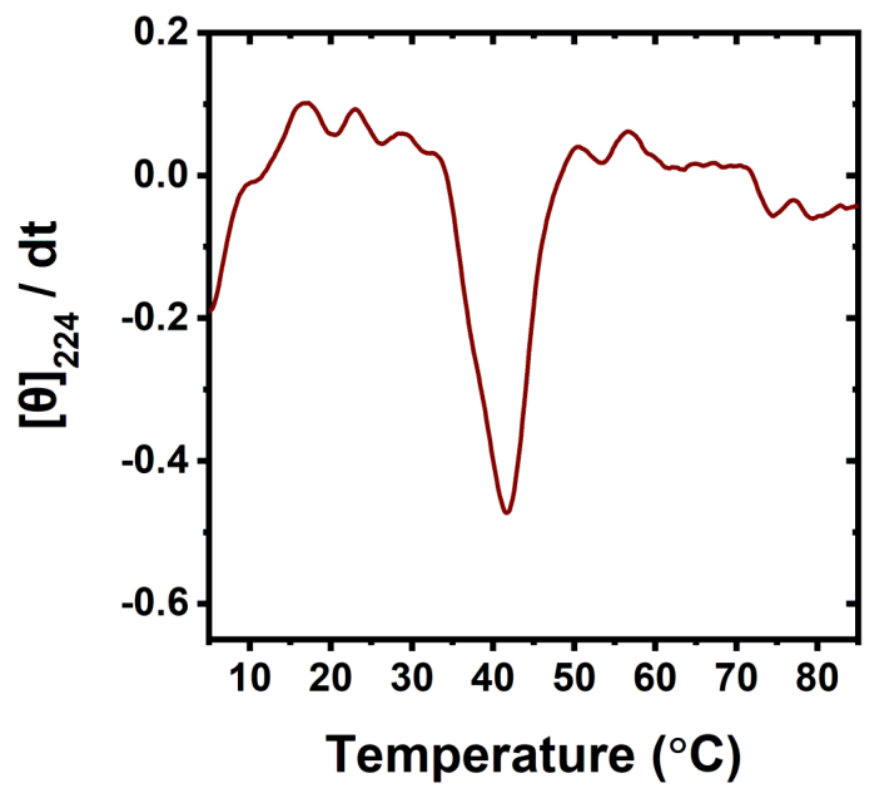

Figure S29. First derivative of the CD signal of $\mathbf{4 R}(\mathbf{X})_{\mathbf{4 4 4}}$ nanosheets $(0.2 \mathrm{mg} / \mathrm{mL})$ assembled in TAPS buffer ( $\mathrm{pH} 8.0$ ) buffer at $224 \mathrm{~nm}$ as a function of temperature. $\mathrm{T}_{\mathrm{m}}=42^{\circ} \mathrm{C}$. 

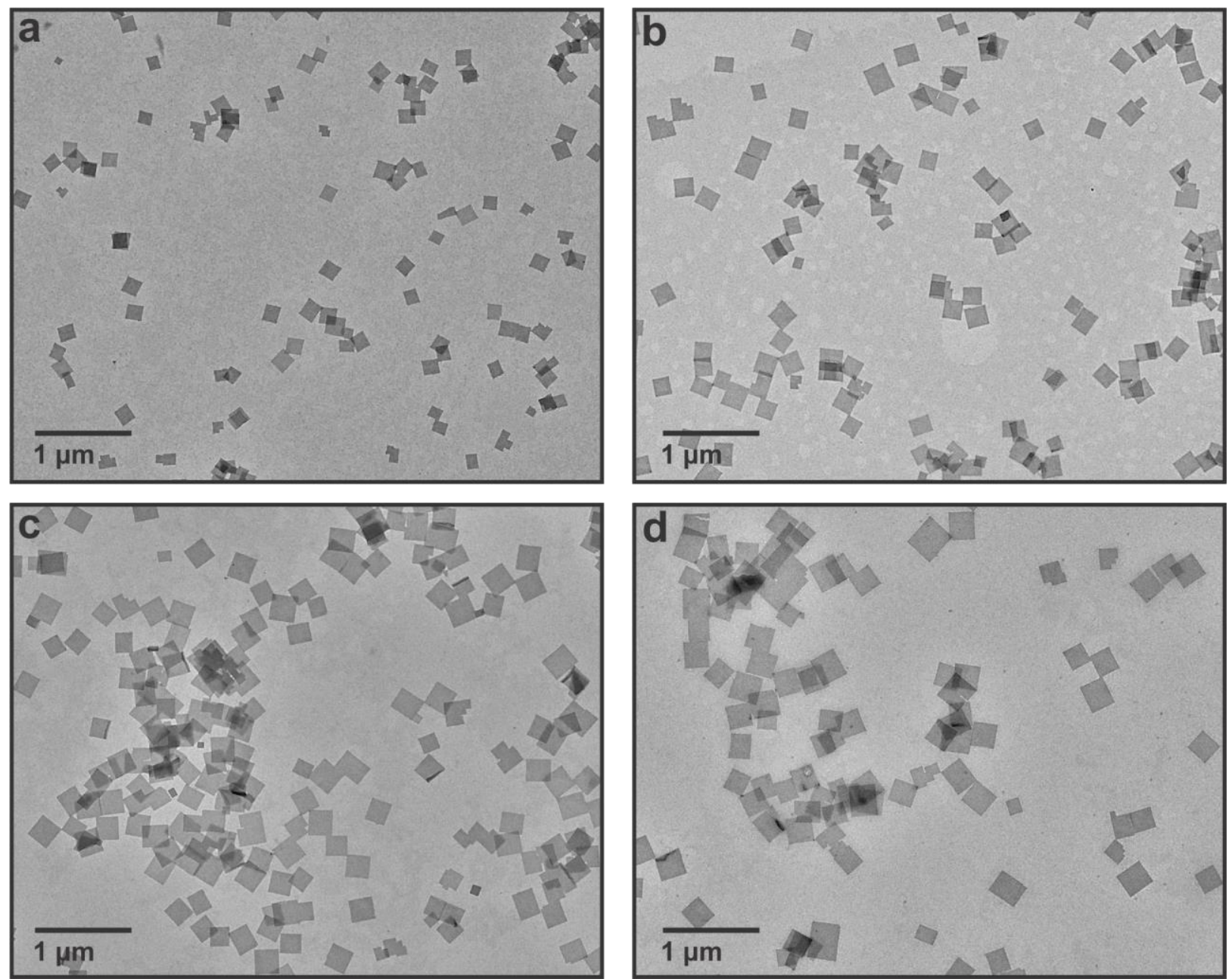

Figure S30. Stained TEM images of $\mathbf{4 R}(X)_{\mathbf{4 4 4}} @ \mathbf{4 S}(X)_{\mathbf{4 4 4}}$ nanosheets assembled in TAPS buffer (pH 8.0) at varying shell/core ratios: (a) 10, (b) 20, (c) 30, and (d) 40. 


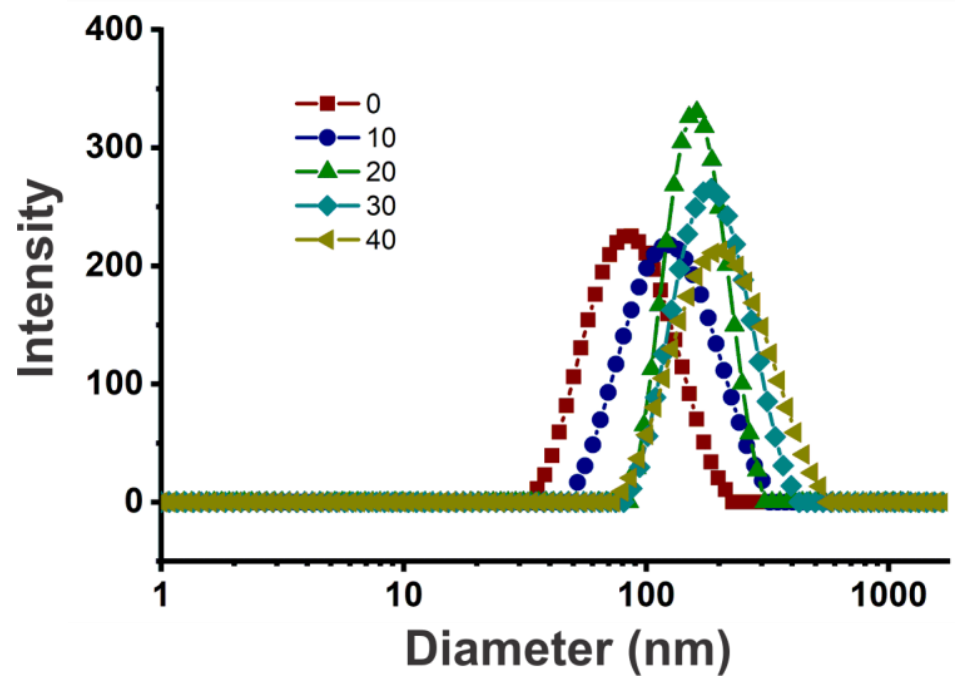

Figure S31. DLS spectra of $\mathbf{4 R}(X)_{444} @ \mathbf{4 S}(X)_{444}$ nanosheets assembled in TAPS buffer (pH 8) with various shell/core ratios employed. DLS spectra were collected after 2 weeks of assembly time. 

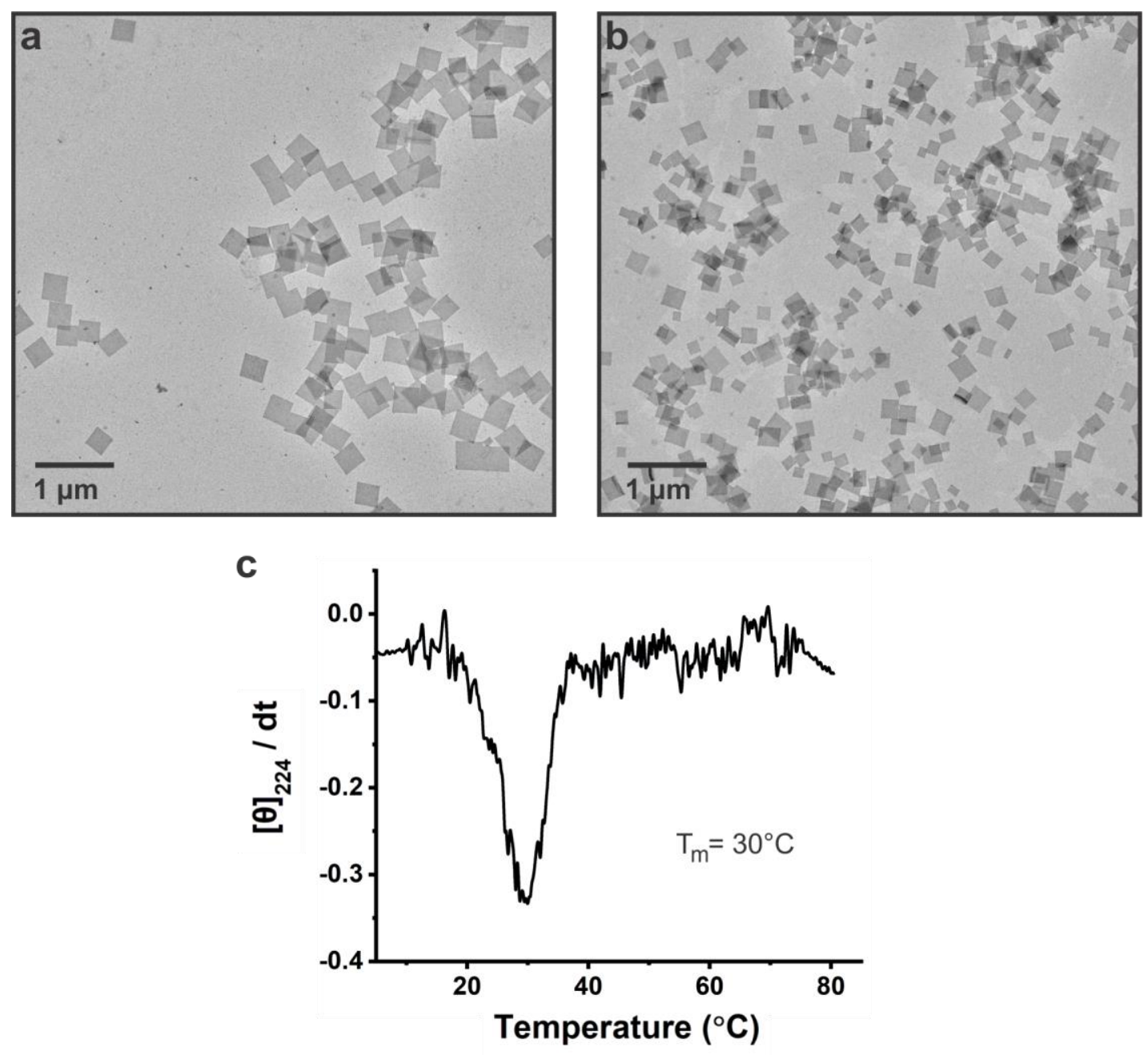

Figure S32. Stained TEM images of $\mathbf{4 S}(\mathbf{X})_{444}$ nanosheets assembled at (a) $2 \mathrm{mg} / \mathrm{mL}$ and (b) 4 $\mathrm{mg} / \mathrm{mL}$ CMP concentration after 2 weeks of assembly time. Nanosheets were assembled in TAPS (pH 8.0) buffer. Doubling the peptide concentration from $2 \mathrm{mg} / \mathrm{mL}$ to $4 \mathrm{mg} / \mathrm{mL}$ decreases nanosheet size, as opposed to nanosheets grown from $4 \mathbf{R}(\mathbf{X})_{444}$ seeds. (c) First derivative of the CD signal of $\mathbf{4 S}(\mathbf{X})_{444}$ nanosheets $(2 \mathrm{mg} / \mathrm{mL})$ assembled in TAPS buffer (pH 8.0) buffer at $224 \mathrm{~nm}$ as a function of temperature. $\mathrm{T}_{\mathrm{m}}=30^{\circ} \mathrm{C}$ 

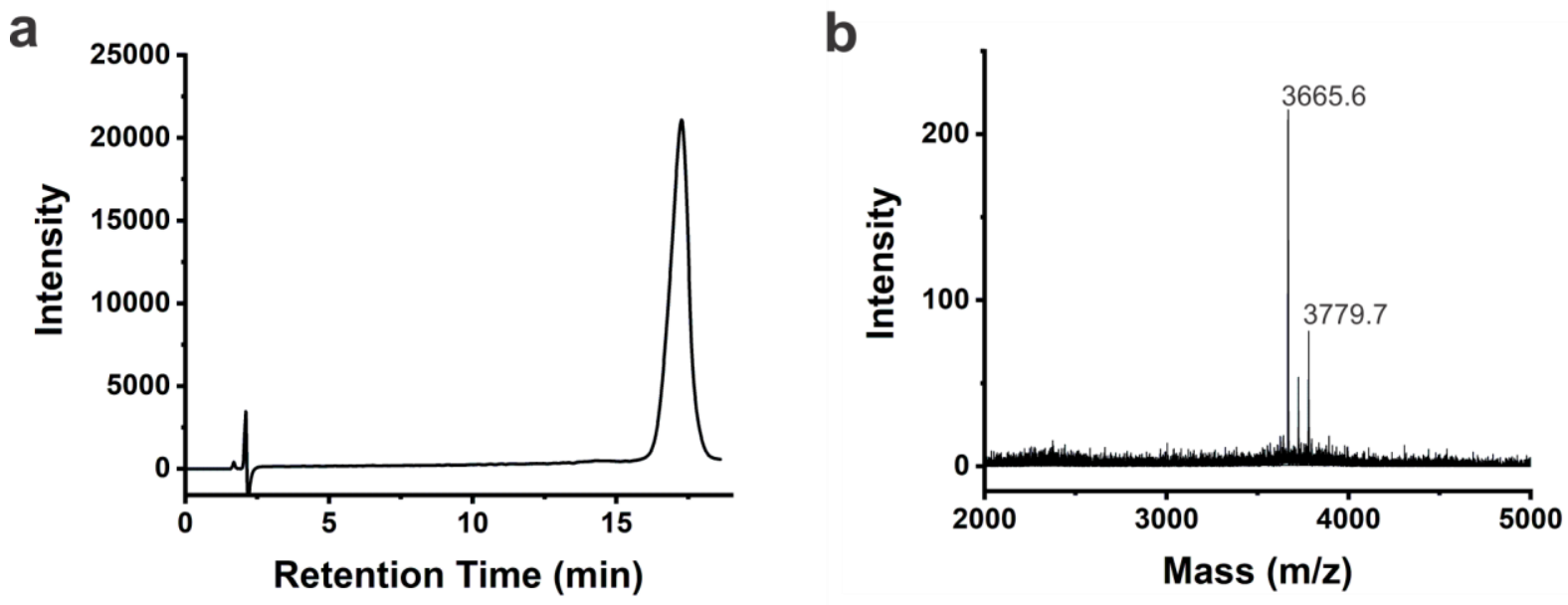

Figure S33. (a) HPLC trace of $\mathbf{N}_{\mathbf{3}} \mathbf{- 4 S}(\mathrm{X})_{\mathbf{4 4 4}}$. (b) MALDI-TOF spectra of $\mathrm{N}_{\mathbf{3}} \mathbf{- 4 R}(\mathrm{X})_{\mathbf{4 4 4}}, \mathrm{m} / \mathrm{z}=$ $3665.6\left(\mathrm{M}+\mathrm{H}^{+}\right), \mathrm{m} / \mathrm{z}=3779.7\left(\mathrm{M}+\mathrm{TFA}^{+}\right)$.
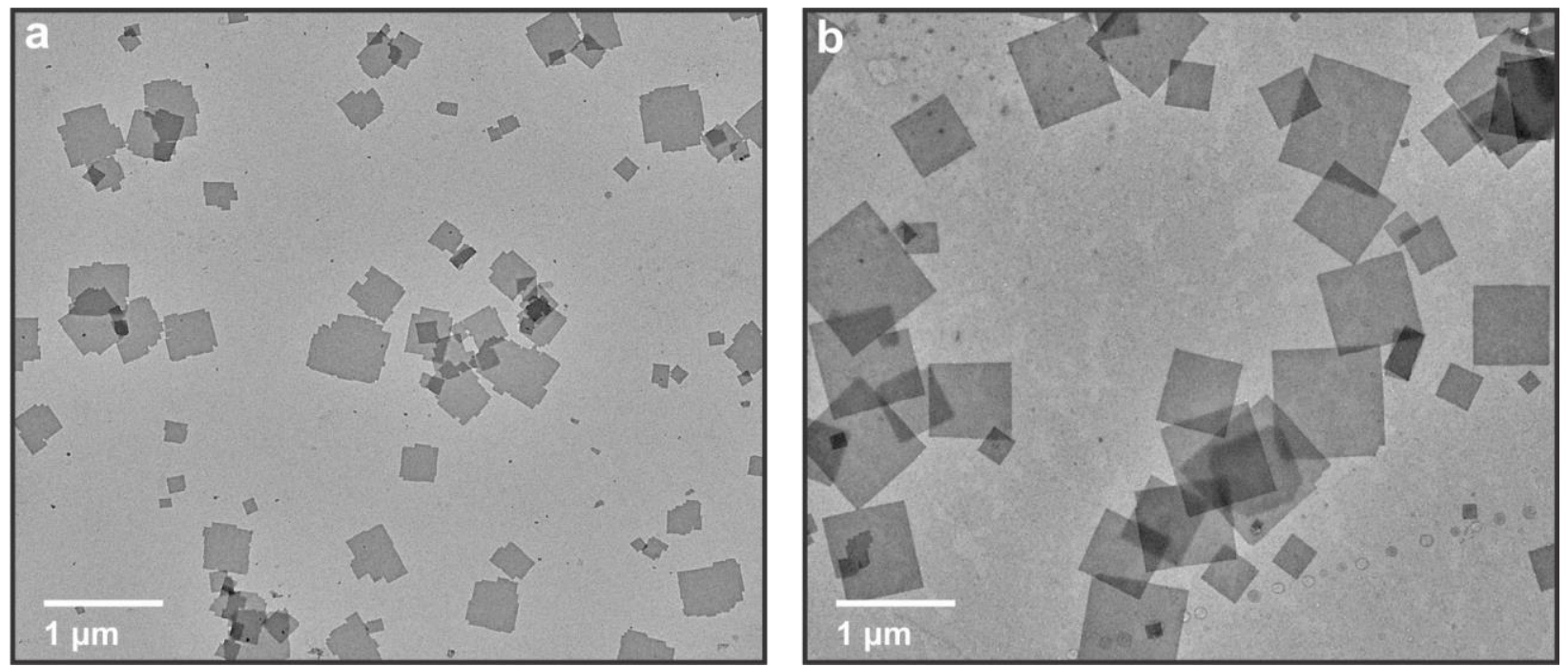

Figure S34. Stained TEM images of (a) b-4R(X)444 nanosheets ( $2 \mathrm{mg} / \mathrm{mL})$ in MOPS pH 7.0 buffer and (b) b-4R(X)444@N3-4S(X)444 nanosheets after 1 week of assembly time. 